\title{
Stability and Change in Fatty Acids Composition of Soybean, Corn, and Sunflower Oils during the Heating Process
}

\author{
Ammar Cherif $\mathbb{D}^{1}$ and Amor Slama $\mathbb{D}^{1,2}$ \\ ${ }^{1}$ University of Tunis El Manar, Faculty of Sciences of Tunis, LR18ES03, Laboratory of Neurophysiology, \\ Cellular Physiopathology and Biomolecules Valorisation, 2092 Tunis, Tunisia \\ ${ }^{2}$ University of Carthage, Faculty of Sciences of Bizerte, Jarzouna-Bizerte 7021, Tunisia \\ Correspondence should be addressed to Ammar Cherif; cherif.ammar@yahoo.com
}

Received 28 August 2021; Revised 27 December 2021; Accepted 5 February 2022; Published 7 March 2022

Academic Editor: Walid Elfalleh

Copyright (c) 2022 Ammar Cherif and Amor Slama. This is an open access article distributed under the Creative Commons Attribution License, which permits unrestricted use, distribution, and reproduction in any medium, provided the original work is properly cited.

\begin{abstract}
This work has been undertaken to investigate the effect of heat treatment on the edible oils (soybean, sunflower, and corn) used in frying and cooking, in particular on the fatty acid composition. The heating process was maintained at $150,180,210$, and $240^{\circ} \mathrm{C}$. At each temperature, the variation of the fatty acid composition was determined after $12,24,36,48$, and $60 \mathrm{~h}$ of treatment by using an improved analytical gas chromatography method. This study showed that the oils, which had undergone a temperature of 150 to $180^{\circ} \mathrm{C}$, kept some thermal stability and preserved their fatty acid composition at different treatment periods. At the temperature $\left(180^{\circ} \mathrm{C}\right)$, two new fatty acids (C8:0 and C12:0) appeared, which could be explained by the transformation of the other fatty acids during the heating process by different chemical reactions. However, the composition of the three oils was significantly affected at $210^{\circ} \mathrm{C}$ and $240^{\circ} \mathrm{C}$. Two trans-fatty acids (C18:1 $9 \mathrm{t}$ and $\left.\mathrm{C} 18: 2 \mathrm{tt}\right)$ were generated proportionally to heat treatment during the heating process for the three oils, providing information on their oxidative state. The results showed that sunflower oil was most affected by the heating temperature than soybean and corn oil. Therefore, more attention should be paid to the heat treatment used and the heating period to preserve the quality of edible oils.
\end{abstract}

\section{Introduction}

Soybean (Glycine max L.), sunflower (Helianthus annuus), and corn (Zea mays L.) oils are edible oils commonly used in frying and baking. The seeds of these plants are rich in fat, mainly in glycerides of unsaturated fatty acids and, in particular, in glycerides of oleic and linoleic acids. The fatty acid composition is often characteristic of each species [1]. The majority of soybean cultivars have around $15-20 \%$ of total lipids [2], corn seeds contain approximately $26.44 \%$ oil [3], and sunflower seeds vary from 31.1 to $52 \%$ oil $[4,5]$. These values are influenced by the weather, geographical edaphic location, agronomic procedures, and oil extraction methods [6]. The other study reported by [7] also revealed that seed metabolite content is sensitive to environmental fluctuations, and the metabolite composition is strongly affected by genotype-environment interactions.
The virtues and benefits of polyunsaturated fatty acids (PUFAs) are multiple. These compounds play an important role in the protection of cardiovascular diseases and have been shown to reduce plasma levels of low-density lipoprotein cholesterol (LDL-C) [8-10]. However, repeated heating of edible oils during frying can damage these benefits and lead to lipid oxidation [11]. Since the lipid components of foods are particularly sensitive to heat treatment, the specific heat fat is low, and they are heated quickly $[12,13]$.

Heat makes many changes to lipids; in fact, heated oil undergoes chemical reactions including oxidation, hydrolysis, polymerization, isomerization, and cyclization. These reactions are detrimental to the stability of fatty acids and other biochemical parameters of oil [14-16]. This may also lead to the production of free fatty acids as well as triglyceride dimers, monounsaturated and diunsaturated fatty acids [17], a transformation of linear fatty acids, and the 
formation of cyclic polymers. In addition, vitamin E, which is a natural antioxidant of oil, also deteriorates after repeated heating. It is well established that heating edible oils causes lipid oxidation [18] and is one of the most undesirable reactions that occur when heating foods. Lipid oxidation causes the destruction of fatty acid chains and affects the nutritional and sensory qualities of foods. A detailed description of the various reactions involved in the oxidation process is broadly described by [19].

Among oxidative damage, free radical formation, which reacts rapidly with oxygen to produce primary and secondary oxidation products, has been frequently observed in vegetable oils. The formation of dimers and polymers decreased the tocopherols [20], and the moderate increase in available fatty acids by the hydrolysis of fats has also been discovered [21-23].

Thus, polyunsaturated fatty acids subjected to prolonged and repeated heat treatments can be transformed into polymers, in trans-fatty acids and cyclic fatty acid monomers (CFAMs) [24-26]. The CFAM content of frying oils, rich in PUFA, can reach 0.02 to $0.7 \%$ of the total fatty acids [27-29]. All these compounds are undesirable in a healthy diet because their harmfulness to health is either known or questioned [30]. The analysis of heating profiles and the associated thermal properties seems to be a valuable tool for assessing the different degrees of thermal oxidation of treated lipids by increasing the temperature of the edible oils and varying the duration of treatment. The study of the oxidative damage of these oils could be faster under heat processing in a conventional oven or fryer treatment $[21,23]$. Lipid oxidation does not only spoil the taste, smell, and color of the oil but may also impose an absolute health threat [31].

Some secondary oxidation products may have a potential risk to the human body, such as volatile compounds (alcohols, aldehydes, ketones, acids, etc.) and nonvolatile compounds (carbonyl or fatty acid dimers, trimers, and cyclic polymers). Volatile compounds may be lost during the frying process; however, nonvolatile polar compounds in the cooking oil are potentially hazardous [32] as they remain in the oil and are then absorbed by food and ingested. In this context, nonvolatile products of lipid oxidation may play an important role in cardiovascular disease. The content of polar compounds in heated oil has been associated with endothelial dysfunction [33] and hypertension [34]. Thus, these products can also cause damage to cell membranes and DNA [35] that may be involved in the aging process and the development of cancer [36].

The main objective of this work is to study the effect of heat treatment on the fatty acid composition of three types of edible oils used in frying and baking. The impact of temperature change and the duration of the heating process on cis/trans, the polyunsaturated fatty acids, and the acquisition of data on lipid oxidation of vegetable oils in specific conditions were also studied. The sample composition of three edible oils was followed during a heat treatment at constant temperatures in an electric furnace and under oxygenation conditions. The temperatures were chosen because they are frequently reached in food during frying and cooking. Samples were analyzed every $12 \mathrm{~h}$ for their total fatty acid composition for $60 \mathrm{~h}$.

The change in the fatty acid composition of the oils studied was determined by gas chromatography. The experimental results were analyzed to show significant differences between the oxidation levels of the oils studied.

\section{Materials and Methods}

2.1. Oil Samples. Three samples of edible oils of soybean, corn, and sunflower purchased from local markets of Tunisia were used in this experiment. Samples were stored in dark vials at $4^{\circ} \mathrm{C}$ before analysis. Each sample was heated at different temperatures in an electric furnace. A total of 63 samples including 1 unheated (control sample) and 20 heated samples for each oil. Pure fatty acid methyl esters (FAMEs) standards used in this study were purchased from Sigma-Aldrich.

2.2. Heating Procedure. Heat treatment was carried out on the three edible oils mentioned above. $150 \mathrm{ml}$ of each oil sample was placed in open beakers and then heated to $150^{\circ} \mathrm{C}$, $180^{\circ} \mathrm{C}, 210^{\circ} \mathrm{C}$, and $240^{\circ} \mathrm{C}$. At each temperature, an oil sample was taken after 12, 24, 36, 48, and 60 hours. The choice of these temperatures was made taking into account the minimum and maximum temperatures, which cover the modes of cooking and frying.

2.3. Fatty Acid Methylation. The fatty acid methylation procedure was carried out in a standardized way to ensure good precision and repeatability of fatty acid analysis. The derivation of fatty acid methyl esters was the most widely used technique for lipid analysis by GC-FID. In fact, $1 \mathrm{ml}$ of hexane and $500 \mu \mathrm{l}$ of sodium methoxide were added to the $10 \mathrm{mg}$ of oil sample. After the vortex, the whole thing was heated between 40 and $50^{\circ} \mathrm{C}$ for $15 \mathrm{~min}$. Then, $4 \mathrm{ml}$ of hexane were added, and $5 \mathrm{ml}$ of $\mathrm{NaCl}$-saturated water were used to wash the sample. Then it was shaken and allowed to stand until the two phases separated. The organic phase was aspirated into a Pasteur pipette containing loose cotton and about $1 \mathrm{mg}$ of dry magnesium sulfate. The filtrate was collected in a test tube.

2.4. Chromatographic Analysis. The chromatographic separation was performed in an autosystem gas chromatograph with a split/splitless injector and a FID detector, equipped with a BPX 70 capillary column of $60 \mathrm{~m}$ length, $0.25 \mathrm{~mm}$ i.d., and $0.25 \mu \mathrm{m}$ film thickness (GC Agilent Technologies, 7890A GC system, Wilmington, USA). The oven temperature was held at $60^{\circ} \mathrm{C}$ for $1 \mathrm{~min}$, and then ramped to $190^{\circ} \mathrm{C}$ at a rate of $10^{\circ} \mathrm{C} / \mathrm{min}$ and maintained for $15 \mathrm{~min}$ before a second ramp at the rate of $5^{\circ} \mathrm{C} / \mathrm{min}$ to $200^{\circ} \mathrm{C}$. It was then held isothermally for $14 \mathrm{~min}$. Hydrogen was used as the carrier gas with a flow rate of $40 \mathrm{ml} / \mathrm{min}$. The temperature of the injector and detector was set at $250^{\circ} \mathrm{C}$. The total flow was $68.7 \mathrm{ml} / \mathrm{min}$ and the pressure was $125.5 / 97.6 \mathrm{kPa}$. The volume injected was $1 \mu \mathrm{L}$, and the analysis time was $45 \mathrm{~min}$. The results are 
expressed as peak area percent. All measurements were performed with three independent replicates.

2.5. Data Analysis. All experiments in this study were performed in triplicate, and the results were expressed as the mean of three replicates \pm the standard deviation (SD).

Let $\Delta C_{i} / C$ be the change rate variation of a fatty acid $\mathrm{C}$ after a heating time $\mathrm{HT}_{i}$ at a given temperature and species. It is defined as follows: $\left(\Delta C_{i} / C\right)=\left(\left|C_{i}-C_{0}\right| / \min \left(C_{i}, C_{0}\right)\right)$ where $\mathrm{C}_{0}$ denotes the value of the fatty acid when no heating is applied.

The data analysis was conducted using ROOT-based scripts [37] (an object-oriented program and library developed by the European Organization for Nuclear Research, or "CERN," for data analysis and plotting, through a dedicated code written in $\mathrm{C}^{++}$) in order to calculate the abovedefined change rate variations of fatty acids. The code runs over the different heating times for each acid, records the maximum change rate variation, and picks up the corresponding parameters. Optimum fatty acid changes could be obtained by comparing the change rate variations over all the species, temperatures, and heating times, as shown in Figures 1-3.

\section{Results and Discussion}

3.1. Untreated Oil. The results obtained by GC-FID suggest the presence of at least 15 fatty acids in fresh soybean and sunflower oils and 16 fatty acids in fresh corn oil (Tables 1-3).

The number of saturated fatty acids (SFAs) was identical for all three oil; for those unsaturated, it was 9 fatty acids in soybean and sunflower oil and 10 for corn oil. However, this number remains variable and depends on the temperature and duration of the treatment (Tables 1 and 4-6).

The study showed that the untreated oils (heating time $=0$ hours) were very rich in unsaturated fatty acids (UFAs), especially oleic acid (C18:1 $\Delta 9 \mathrm{C})$ and linoleic (C18: $2 n-6)$. The rate of these two fatty acids together was 76.19 , 81.07 , and $84.36 \%$, respectively, in soybean, sunflower, and corn oil. However, linolenic acid content was only $0.72,5.12$, and $6.25 \%$ in corn, sunflower, and soybean oil, respectively (Tables 1 and 4-6). These results show that these oils are important natural sources of unsaturated fatty acids of omega 6, but that these samples are poor in essential fatty acids of omega-3. Sunflower oil was the richest in omega- 6 unsaturated fatty acids, followed by soybean oil. It was necessary to note the presence of minor fatty acids was indicated in the previous tables (less than $2 \%$ in fresh oils) but were not identified.

3.2. Effect of Treatment at $150^{\circ} \mathrm{C}$. Palmitic acid levels gradually increased with the duration of treatment and ranged from 10.33 to $11.08 \%$ after 60 hours of heat treatment for soybean and from 6.29 to $6.70 \%$ for sunflower and from 10.28 to $12.56 \%$ for corn oil. C18:1 11C increased slightly; however, the results show a slight decrease in the levels of

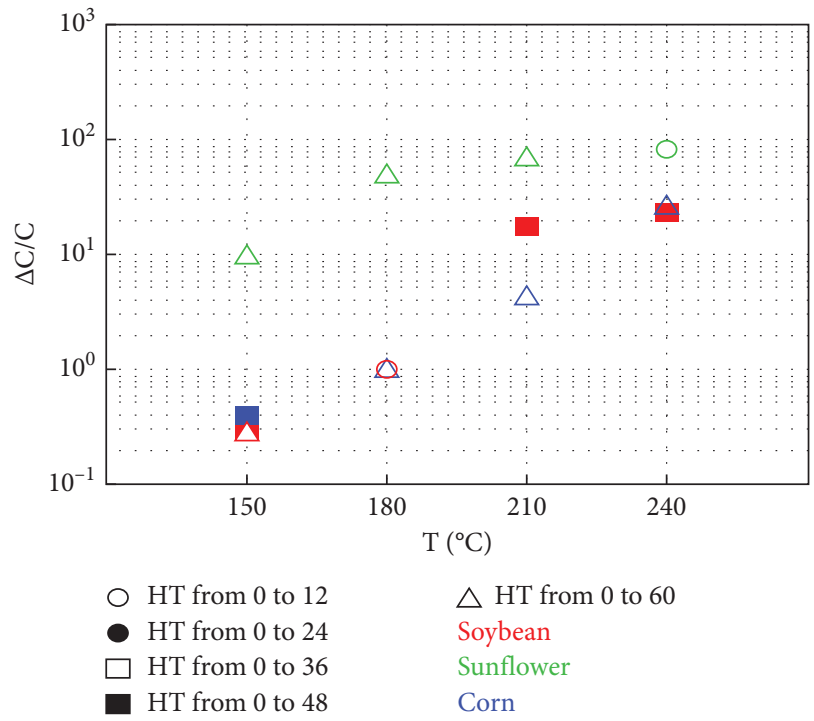

FIGURE 1: Effect of the temperature on the maximum change rate of fatty acids over all the three species (soybean, sunflower, and corn). The heating times (HT) are represented as supplementary explanatory variables.

essential fatty acids (C18: $2 n-6$ and C18: $3 n-3)$ for soybean and a significant decrease in C18: $3 n-3$ of sunflower depending on the duration of treatment. The sharp reduction of C18: $3 n-3$ after the first heating $\left(150^{\circ} \mathrm{C}\right)$ showed that sunflower oil was more sensitive compared to the other oils. In fact, the transformation of $\mathrm{C} 18: 3 n-3$ from 5.12 to $0.48 \%$ after $12 \mathrm{~h}$ of treatment suggests that sunflower oil contains the least antioxidant compounds, including tocopherols, than other oils, and it is, therefore, the most exposed to oxidation. In contrast, most other fatty acids were stable in all three oils (Table 2). In untreated oils, trans-fatty acid (TFA) (C18: 19 t) was found only in corn oil and remained almost stable during the treatment process at $150^{\circ} \mathrm{C}$. In sunflower and soybean oils, C18: $19 \mathrm{t}$ appeared after 24 and 60 hours of heating.

3.3. Effect of Treatment at $180^{\circ} \mathrm{C}$. At this temperature, there is the emergence of new compounds. In effect, C12: 0 appeared during all stages (12, 24, 36, 48, and 60 hours) and C8: 0 at the last two stages (48 and 60 hours) of heat treatment of soybean oil. However, in sunflower, C8: 0 appeared in the last three stages (36, 48, and 60 hours) and in corn only in the last stage (60 hours) (Tables 4, 7, and 8). Some lower molecular weight compounds have been reported to form under severe heating conditions [38]. Data from the literature also show that oil rich in unsaturated fatty acids with many carbon-carbon double bonds can be converted into other reactive groups [39]. C18: $19 \mathrm{t}$ began to appear in the early stages of heat treatment $(12 \mathrm{~h})$ for soybean and sunflower oils. Despite the appearance of new compounds indicating the onset of chemical transformation reactions, there was thermal stability of the fatty acid composition at all stages and in all three oils. 


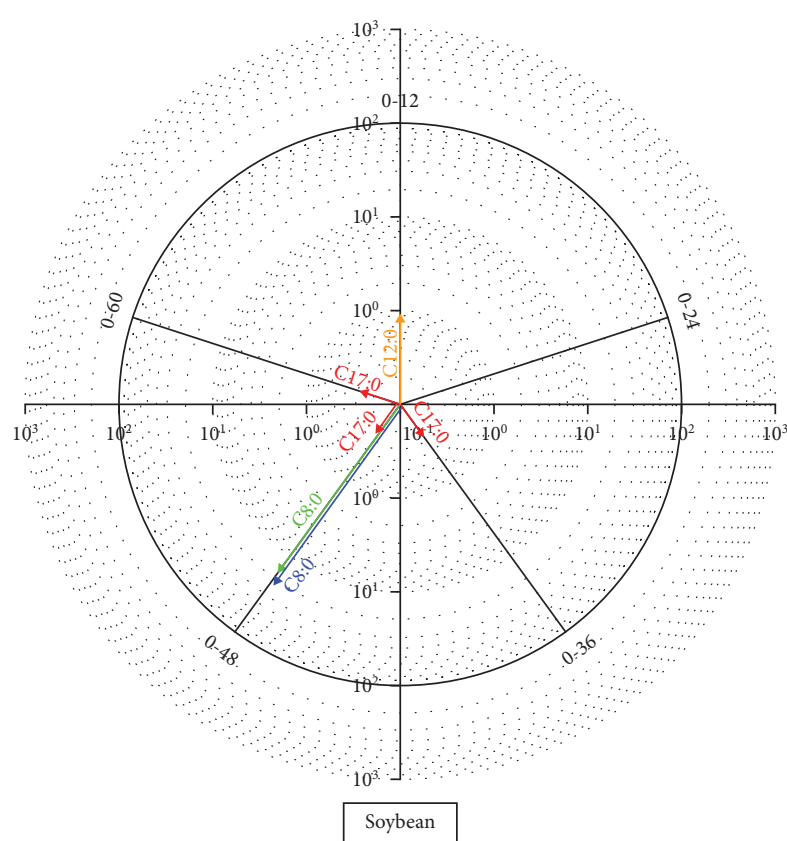

$\mathrm{T}=150^{\circ} \mathrm{C}$ $\mathrm{T}=180^{\circ} \mathrm{C}$ $\begin{aligned} \mathrm{T} & =210^{\circ} \mathrm{C} \\ \mathrm{T} & =240^{\circ} \mathrm{C}\end{aligned}$

(a)

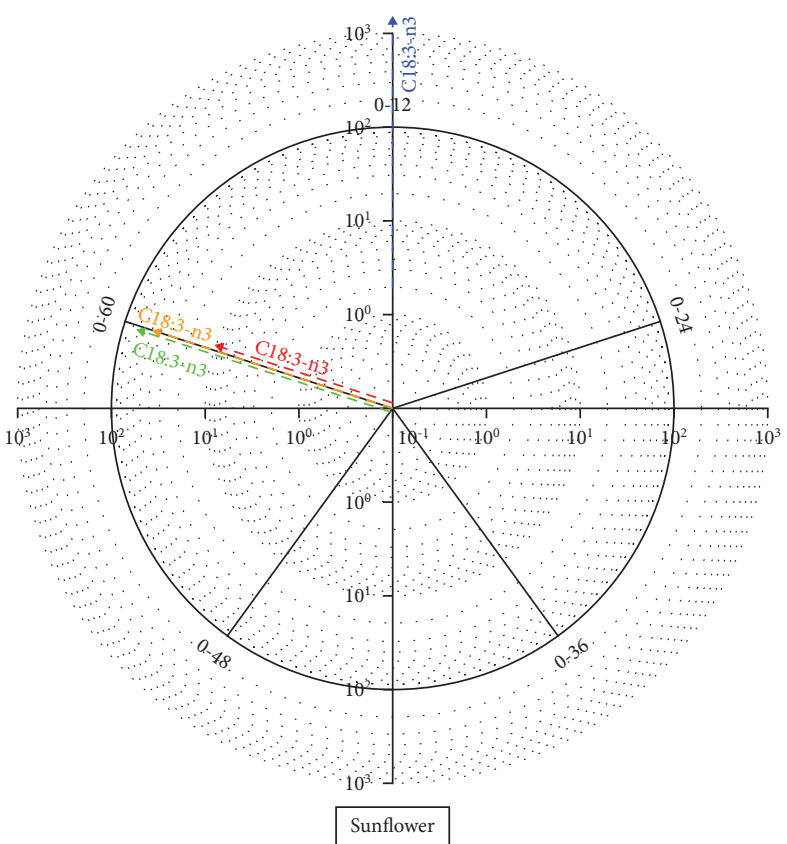

$\mathrm{T}=150{ }^{\circ} \mathrm{C}$

$\mathrm{T}=180^{\circ} \mathrm{C}$

$\mathrm{T}=210^{\circ} \mathrm{C}$

$\mathrm{T}=240^{\circ} \mathrm{C}$

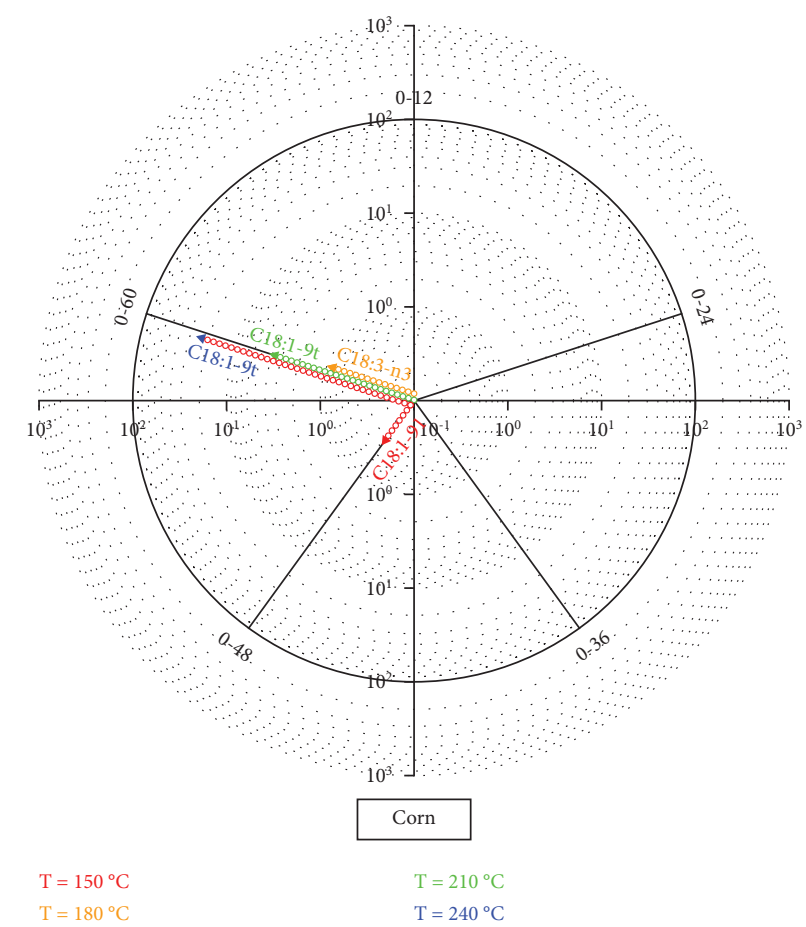

(c)

Figure 2: Heating time variations (in hours) leading to maximum change rate of fatty acids at different temperatures per species: (a) soybean, (b) sunflower, and (c) corn. The vector length and direction designate the change rate of the fatty acid and the heating time variation, respectively. The fatty acid compositions are written as supplementary explanatory variables.

3.4. Effect of Treatment at $210^{\circ} \mathrm{C}$. The two new fatty acids (C8: 0 and C12: 0) mentioned above were present in all stages of the heat treatment of soybean oil. C8: 0 was present in all stages of sunflower oil heat treatment (Table 5) and only in the final stage of processing $(60 \mathrm{~h})$ of corn oil. Levels of major saturated fatty acids (SFAs), including C16: 0 and C18: 0, continued to increase at $210^{\circ} \mathrm{C}$ and varied in the start of treatment $(12 \mathrm{~h})$, from 10.33 to $12.28 \%$ and from 3.53 to 

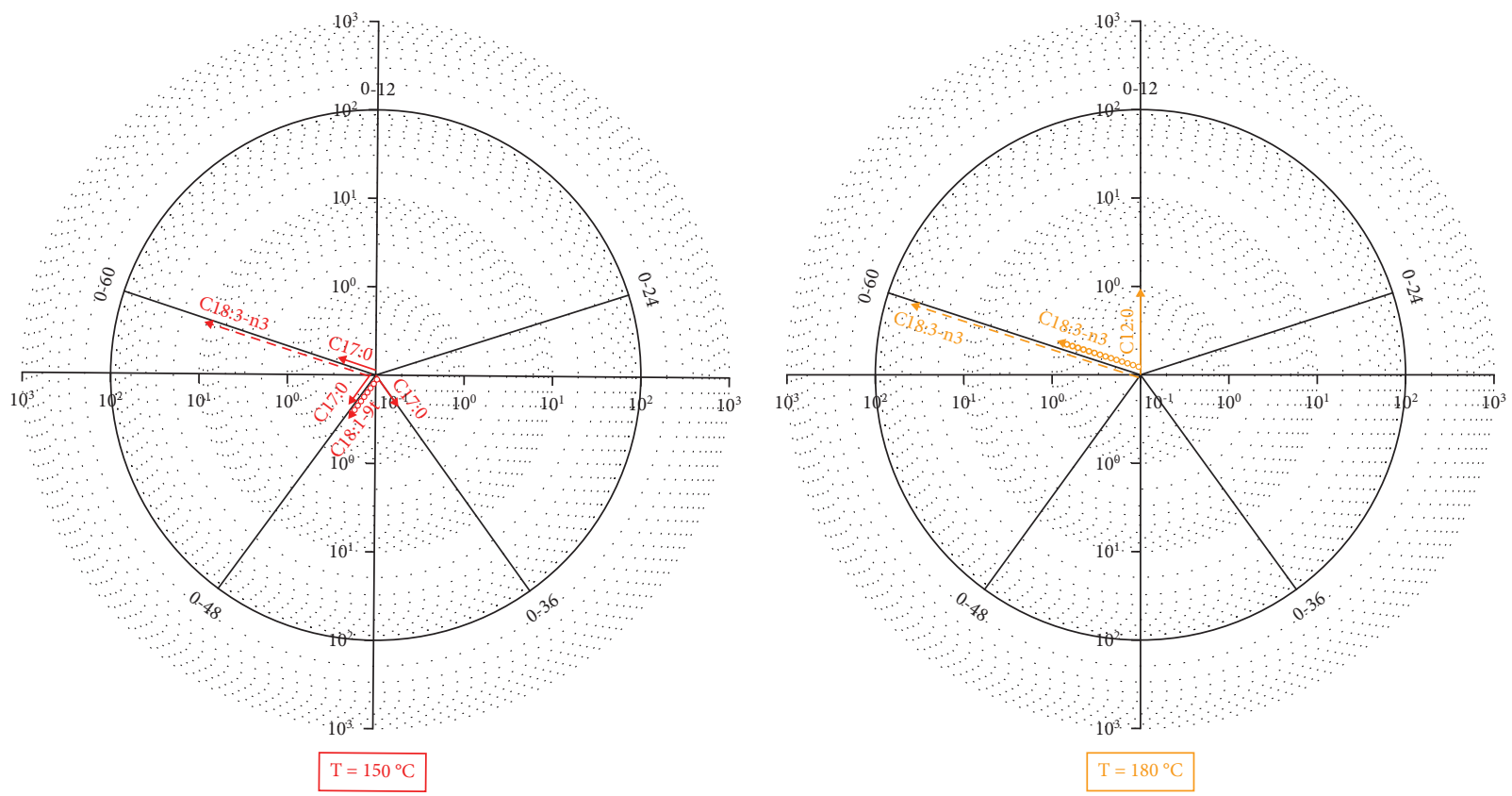

$$
\begin{array}{ll}
\longrightarrow & \text { Soybean } \\
\longrightarrow & \text { Sunflower } \\
\text { cosocos } & \text { Corn }
\end{array}
$$

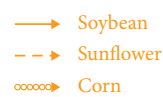

(a)
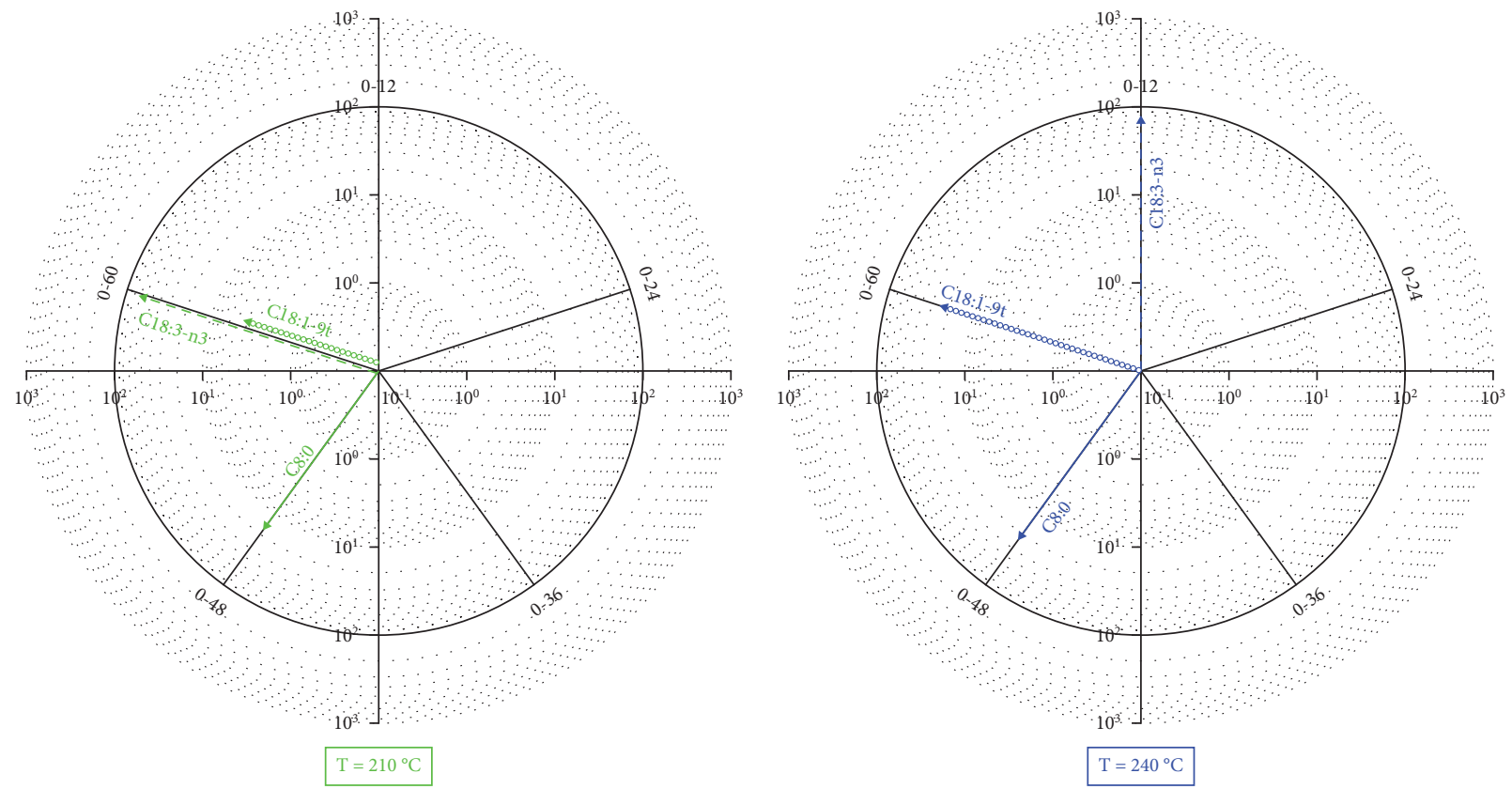

$$
\begin{array}{ll}
\longrightarrow & \text { Soybean } \\
\longrightarrow \rightarrow & \text { Sunflower } \\
\infty & \text { Corn }
\end{array}
$$

$$
\begin{array}{ll}
\longrightarrow & \text { Soybean } \\
\longrightarrow & \text { Sunflower } \\
000000 \text { Corn }
\end{array}
$$

(c)

(d)

FIgURE 3: Heating time variations (in hours) leading to maximum change rate of fatty acids over all the three species at different temperatures: (a) at $150^{\circ} \mathrm{C},(\mathrm{b})$ at $180^{\circ} \mathrm{C}$, (c) at $210^{\circ} \mathrm{C}$, and (d) at $240^{\circ} \mathrm{C}$. The vector length and direction designate the change rate of the fatty acid and the heating time variation, respectively. The fatty acid compositions are written as supplementary explanatory variables. 
TABLe 1: Variation of fatty acids of soybean oil under $150^{\circ} \mathrm{C}$ heating temperature at different heating times.

\begin{tabular}{|c|c|c|c|c|c|c|}
\hline \multicolumn{7}{|c|}{ Heating time (hours)/fatty acids (\%) } \\
\hline & $0^{*}$ & 12 & 24 & 36 & 48 & 60 \\
\hline C8:0 & - & - & - & - & - & - \\
\hline $\mathrm{C} 12: 0$ & - & - & - & - & - & - \\
\hline C14:0 & $0.05 \pm 0.01$ & $0.06 \pm 0.01$ & $0.06 \pm 0$ & $0.06 \pm 0$ & $0.06 \pm 0$ & $0.06 \pm 0$ \\
\hline $\mathrm{C} 16: 0$ & $10.33 \pm 0.12$ & $10.45 \pm 0.04$ & $10.52 \pm 0.04$ & $10.76 \pm 0.09$ & $10.81 \pm 0.03$ & $11.08 \pm 0.01$ \\
\hline C16:1 & $0.06 \pm 0$ & $0.06 \pm 0.01$ & $0.06 \pm 0$ & $0.06 \pm 0$ & $0.06 \pm 0$ & $0.06 \pm 0$ \\
\hline $\mathrm{C} 17: 0$ & $0.07 \pm 0$ & $0.08 \pm 0.01$ & $0.08 \pm 0$ & $0.09 \pm 0.01$ & $0.09 \pm 0$ & $0.09 \pm 0$ \\
\hline $\mathrm{C} 17: 1$ & $0.02 \pm 0.01$ & $0.03 \pm 0.01$ & $0.02 \pm 0.02$ & $0.03 \pm 0$ & $0.03 \pm 0$ & $0.03 \pm 0$ \\
\hline C18:0 & $3.53 \pm 0.03$ & $3.29 \pm 0.02$ & $3.32 \pm 0.02$ & $3.39 \pm 0.05$ & $3.42 \pm 0.02$ & $3.51 \pm 0$ \\
\hline C18:1 9t & - & - & - & - & - & $0.04 \pm 0$ \\
\hline C18:1 9c & $21.23 \pm 0.14$ & $20.33 \pm 0.08$ & $20.43 \pm 0.08$ & $20.77 \pm 0.08$ & $20.81 \pm 0.03$ & $21.16 \pm 0.02$ \\
\hline C18:1 11c & $1.1 \pm 0.02$ & $1.03 \pm 0.01$ & $1.04 \pm 0$ & $1.06 \pm 0.02$ & $1.07 \pm 0.01$ & $1.08 \pm 0.01$ \\
\hline $\mathrm{C} 18: 2 \mathrm{tt}$ & - & - & - & - & - & - \\
\hline $\mathrm{C} 18: 2 n-6$ & $54.96 \pm 0.36$ & $56.01 \pm 0.31$ & $55.94 \pm 0.35$ & $55.61 \pm 0.2$ & $55.49 \pm 0.11$ & $54.86 \pm 0.07$ \\
\hline $\mathrm{C} 18: 3 n-6$ & $0.44 \pm 0.01$ & $0.5 \pm 0.01$ & $0.49 \pm 0.01$ & $0.48 \pm 0$ & $0.48 \pm 0$ & $0.46 \pm 0$ \\
\hline $\mathrm{C} 18: 3 n-3$ & $6.25 \pm 0.06$ & $6.22 \pm 0.05$ & $6.12 \pm 0.04$ & $5.94 \pm 0.07$ & $5.94 \pm 0.07$ & $5.73 \pm 0.01$ \\
\hline C20:0 & $0.26 \pm 0.01$ & $0.23 \pm 0.01$ & $0.24 \pm 0$ & $0.24 \pm 0.01$ & $0.24 \pm 0.01$ & $0.25 \pm 0$ \\
\hline C20:1 & $0.15 \pm 0$ & $0.14 \pm 0.01$ & $0.14 \pm 0$ & $0.14 \pm 0$ & $0.14 \pm 0$ & $0.14 \pm 0.01$ \\
\hline C22:0 & $0.3 \pm 0.02$ & $0.26 \pm 0.01$ & $0.26 \pm 0$ & $0.26 \pm 0.01$ & $0.27 \pm 0.01$ & $0.26 \pm 0$ \\
\hline Other FAs & 2.39 & 1.32 & 1.28 & 1.11 & 1.09 & 1.18 \\
\hline$\Sigma$ SFA & 14.54 & 14.37 & 14.47 & 14.81 & 14.89 & 15.25 \\
\hline$\Sigma$ UFA & 83.07 & 84.32 & 84.24 & 84.09 & 84.02 & 83.56 \\
\hline$\Sigma$ TFA & - & - & - & - & - & 0.04 \\
\hline
\end{tabular}

TABLE 2: Variation of fatty acids of sunflower oil under $150^{\circ} \mathrm{C}$ heating temperature at different heating times.

\begin{tabular}{|c|c|c|c|c|c|c|}
\hline \multicolumn{7}{|c|}{ Heating time (hours)/fatty acids (\%) } \\
\hline & $0^{*}$ & 12 & 24 & 36 & 48 & 60 \\
\hline C8:0 & - & - & - & - & - & $0.06 \pm 0.01$ \\
\hline $\mathrm{C} 12: 0$ & - & - & - & - & - & - \\
\hline C14:0 & $0.05 \pm 0.01$ & $0.05 \pm 0.01$ & $0.05 \pm 0.01$ & $0.05 \pm 0.01$ & $0.05 \pm 0.01$ & $0.06 \pm 0.01$ \\
\hline $\mathrm{C} 16: 0$ & $6.29 \pm 0.18$ & $6.42 \pm 0.03$ & $6.36 \pm 0.01$ & $6.41 \pm 0.02$ & $6.52 \pm 0.02$ & $6.7 \pm 0.05$ \\
\hline $\mathrm{C} 16: 1$ & $0.06 \pm 0.01$ & $0.07 \pm 0.01$ & $0.07 \pm 0.01$ & $0.07 \pm 0.01$ & $0.07 \pm 0.01$ & $0.07 \pm 0.01$ \\
\hline C17:0 & $0.07 \pm 0.01$ & $0.03 \pm 0.01$ & $0.03 \pm 0.01$ & $0.03 \pm 0.01$ & $0.04 \pm 0.01$ & $0.04 \pm 0.01$ \\
\hline C17:1 & - & - & - & - & - & - \\
\hline C18:0 & $3.44 \pm 0.19$ & $3.22 \pm 0.02$ & $3.22 \pm 0.02$ & $3.25 \pm 0.01$ & $3.3 \pm 0.02$ & $3.36 \pm 0.02$ \\
\hline C18:1 9t & - & - & $0.05 \pm 0.01$ & $0.06 \pm 0.01$ & $0.05 \pm 0.01$ & $0.06 \pm 0.02$ \\
\hline $\mathrm{C} 18: 19 \mathrm{c}$ & $25.54 \pm 0.13$ & $24.37 \pm 0.02$ & $24.39 \pm 0.02$ & $24.54 \pm 0.07$ & $24.81 \pm 0.01$ & $25.16 \pm 0.19$ \\
\hline $\mathrm{C} 18: 1 \mathrm{11c}$ & $1.29 \pm 0.33$ & $0.53 \pm 0.01$ & $0.54 \pm 0.01$ & $0.54 \pm 0.01$ & $0.55 \pm 0.01$ & $0.56 \pm 0.01$ \\
\hline $\mathrm{C} 18: 2 \mathrm{tt}$ & - & - & - & - & - & - \\
\hline $\mathrm{C} 18: 2 n-6$ & $55.53 \pm 0.95$ & $63.06 \pm 0.07$ & $62.89 \pm 0.12$ & $62.4 \pm 0.32$ & $61.9 \pm 0.14$ & $60.98 \pm 0.54$ \\
\hline $\mathrm{C} 18: 3 n-6$ & $0.43 \pm 0.03$ & - & - & - & - & - \\
\hline $\mathrm{C} 18: 3 n-3$ & $5.12 \pm 0.03$ & $0.48 \pm 0.02$ & $0.48 \pm 0.03$ & $0.47 \pm 0.04$ & $0.46 \pm 0.04$ & $0.45 \pm 0.03$ \\
\hline $\mathrm{C} 20: 0$ & $0.25 \pm 0.03$ & $0.2 \pm 0.01$ & $0.19 \pm 0.01$ & $0.2 \pm 0.01$ & $0.2 \pm 0.01$ & $0.21 \pm 0.01$ \\
\hline C20:1 & $0.14 \pm 0.02$ & $0.11 \pm 0.01$ & $0.1 \pm 0.01$ & $0.1 \pm 0.01$ & $0.1 \pm 0.01$ & $0.1 \pm 0.01$ \\
\hline C22:0 & $0.27 \pm 0.02$ & $0.52 \pm 0.01$ & $0.51 \pm 0.01$ & $0.52 \pm 0.01$ & $0.54 \pm 0.01$ & $0.53 \pm 0.01$ \\
\hline Other FAs & 1.52 & 0.95 & 1.11 & 1.36 & 1.41 & 1.73 \\
\hline$\Sigma$ SFA & 10.37 & 10.44 & 10.38 & 10.46 & 10.65 & 10.89 \\
\hline$\Sigma$ UFA & 88.11 & 88.61 & 88.51 & 88.18 & 87.94 & 87.38 \\
\hline$\Sigma \mathrm{TFA}$ & - & - & 0.05 & 0.06 & 0.05 & 0.06 \\
\hline
\end{tabular}

$4.08 \%$, respectively, after $60 \mathrm{~h}$ of processing of soybean oil. Minor saturated fatty acids also show a gradual increase as the duration of treatment increases. However, the content of essential fatty acids decreased over the processing time from $54.96 \%$ to $51.25 \%$ for C18: $2 n-6$ and from $6.25 \%$ to $3.74 \%$ for C18: $3 n-3$ in soybean oil (Table 5).
At the same time and in the same type of oil, there was an increase in the C18: $19 \mathrm{c}$ content (from $21.23 \%$ to $23.64 \%$ ) and C18: $3 n-6$ (from $0.44 \%$ to $0.49 \%$ ). For trans-fatty acids, there was a significant increase in the C18: $19 \mathrm{t}$ level at the end of the processing process that was more than 4 times for sunflower and corn oils and 11 times for soybean oil 
TABLE 3: Variation of fatty acids of corn oil under $150^{\circ} \mathrm{C}$ heating temperature at different heating times.

\begin{tabular}{|c|c|c|c|c|c|c|}
\hline \multicolumn{7}{|c|}{ Heating time (hours)/fatty acids (\%) } \\
\hline & $0^{*}$ & 12 & 24 & 36 & 48 & 60 \\
\hline C8:0 & - & - & - & - & - & - \\
\hline C12:0 & - & - & - & - & - & - \\
\hline C14:0 & $0.02 \pm 0.01$ & $0.02 \pm 0.01$ & $0.02 \pm 0.01$ & $0.02 \pm 0.01$ & $0.02 \pm 0.01$ & $0.02 \pm 0.01$ \\
\hline C16:0 & $10.28 \pm 0.11$ & $12.26 \pm 0.03$ & $12.27 \pm 0.06$ & $12.32 \pm 0.07$ & $12.34 \pm 0.14$ & $12.56 \pm 0.08$ \\
\hline C16:1t & $0.03 \pm 0$ & $0.03 \pm 0.01$ & $0.03 \pm 0.01$ & $0.03 \pm 0.01$ & $0.03 \pm 0.01$ & $0.03 \pm 0.01$ \\
\hline $\mathrm{C} 16: 1 \mathrm{c}$ & $0.08 \pm 0$ & $0.1 \pm 0.01$ & $0.1 \pm 0.01$ & $0.1 \pm 0.01$ & $0.1 \pm 0.01$ & $0.1 \pm 0.01$ \\
\hline $\mathrm{C} 17: 0$ & $0.05 \pm 0.01$ & $0.06 \pm 0.01$ & $0.06 \pm 0.01$ & $0.06 \pm 0.01$ & $0.06 \pm 0.01$ & $0.06 \pm 0.01$ \\
\hline $\mathrm{C} 17: 1$ & $0.02 \pm 0.01$ & $0.03 \pm 0.01$ & $0.02 \pm 0.01$ & $0.02 \pm 0.01$ & $0.02 \pm 0.01$ & $0.03 \pm 0.01$ \\
\hline C18:0 & $1.49 \pm 0.02$ & $1.8 \pm 0.01$ & $1.8 \pm 0.02$ & $1.81 \pm 0.01$ & $1.81 \pm 0.02$ & $1.83 \pm 0.01$ \\
\hline C18:1 9t & $0.05 \pm 0.01$ & $0.04 \pm 0.02$ & $0.05 \pm 0.02$ & $0.06 \pm 0.02$ & $0.07 \pm 0.03$ & $0.05 \pm 0.02$ \\
\hline $\mathrm{C} 18: 19 \mathrm{c}$ & $30.7 \pm 0.27$ & $34.63 \pm 0.19$ & $34.65 \pm 0.11$ & $34.77 \pm 0.26$ & $34.78 \pm 0.4$ & $35.21 \pm 0.27$ \\
\hline C18:1 11c & $0.47 \pm 0.01$ & $0.47 \pm 0.01$ & $0.47 \pm 0.01$ & $0.47 \pm 0.01$ & $0.47 \pm 0.01$ & $0.47 \pm 0.01$ \\
\hline $\mathrm{C} 18: 2 \mathrm{tt}$ & - & - & - & - & - & - \\
\hline $\mathrm{C} 18: 2 n-6$ & $53.66 \pm 0.13$ & $48.24 \pm 0.29$ & $48.14 \pm 0.14$ & $47.87 \pm 0.36$ & $47.37 \pm 0.55$ & $47.09 \pm 0.39$ \\
\hline C18:3 $n-6$ & $0.05 \pm 0.01$ & - & - & - & - & - \\
\hline C18:3 $n-3$ & $0.72 \pm 0.01$ & $0.65 \pm 0.04$ & $0.63 \pm 0.03$ & $0.66 \pm 0.04$ & $0.66 \pm 0.05$ & $0.59 \pm 0.03$ \\
\hline C20:0 & $0.38 \pm 0.01$ & $0.4 \pm 0.01$ & $0.4 \pm 0.01$ & $0.4 \pm 0.01$ & $0.4 \pm 0.01$ & $0.41 \pm 0.01$ \\
\hline C20:1 & $0.2 \pm 0.01$ & $0.18 \pm 0.01$ & $0.18 \pm 0.01$ & $0.18 \pm 0.01$ & $0.18 \pm 0.01$ & $0.19 \pm 0.01$ \\
\hline $\mathrm{C} 22: 0$ & $0.09 \pm 0.01$ & $0.08 \pm 0.01$ & $0.08 \pm 0$ & $0.08 \pm 0.03$ & $0.1 \pm 0.03$ & $0.1 \pm 0.02$ \\
\hline Other FAs & 1.71 & 1.02 & 1.12 & 1.15 & 1.58 & 1.33 \\
\hline$\Sigma$ SFA & 12.31 & 14.63 & 14.62 & 13.85 & 14.74 & 14.40 \\
\hline$\Sigma \mathrm{UFA}$ & 85.98 & 84.35 & 84.26 & 84.85 & 83.69 & 84.26 \\
\hline$\Sigma$ TFA & 0.08 & 0.07 & 0.08 & 0.09 & 0.11 & 0.08 \\
\hline
\end{tabular}

FA: fatty acid; SFA: saturated fatty acid; UFA: unsaturated fatty acid; TFA: trans-fatty acid. —: not detected; ${ }^{*}$ : fresh oil.

TABLE 4: Variation of fatty acids of soybean oil under $180^{\circ} \mathrm{C}$ heating temperature at different heating times.

\begin{tabular}{|c|c|c|c|c|c|c|}
\hline \multicolumn{7}{|c|}{ Heating time (hours)/fatty acids (\%) } \\
\hline & $0^{*}$ & 12 & 24 & 36 & 48 & 60 \\
\hline C8:0 & - & - & - & - & $0.06 \pm 0.01$ & $0.1 \pm 0.01$ \\
\hline $\mathrm{C} 12: 0$ & - & $0.02 \pm 0$ & $0.01 \pm 0.01$ & $0.02 \pm 0$ & $0.02 \pm 0$ & $0.02 \pm 0.01$ \\
\hline C14:0 & $0.05 \pm 0.01$ & $0.06 \pm 0.01$ & $0.06 \pm 0$ & $0.07 \pm 0.01$ & $0.07 \pm 0$ & $0.07 \pm 0$ \\
\hline $\mathrm{C} 16: 0$ & $10.33 \pm 0.12$ & $10.39 \pm 0$ & $10.45 \pm 0.07$ & $10.7 \pm 0.01$ & $11.01 \pm 0.03$ & $11.51 \pm 0.01$ \\
\hline C16:1 & $0.06 \pm 0$ & $0.06 \pm 0$ & $0.06 \pm 0$ & $0.06 \pm 0$ & $0.06 \pm 0$ & $0.07 \pm 0$ \\
\hline $\mathrm{C} 17: 0$ & $0.07 \pm 0$ & $0.08 \pm 0$ & $0.08 \pm 0$ & $0.08 \pm 0$ & $0.09 \pm 0.01$ & $0.1 \pm 0$ \\
\hline $\mathrm{C} 17: 1$ & $0.02 \pm 0.01$ & $0.03 \pm 0.01$ & $0.03 \pm 0$ & $0.03 \pm 0$ & $0.03 \pm 0$ & $0.03 \pm 0$ \\
\hline C18:0 & $3.53 \pm 0.03$ & $3.4 \pm 0.01$ & $3.43 \pm 0.03$ & $3.49 \pm 0.02$ & $3.6 \pm 0.02$ & $3.76 \pm 0.02$ \\
\hline $\mathrm{C} 18: 19 \mathrm{t}$ & & $0.05 \pm 0.02$ & $0.05 \pm 0.01$ & $0.04 \pm 0.01$ & $0.05 \pm 0.02$ & $0.08 \pm 0.02$ \\
\hline C18:1 9c & $21.23 \pm 0.14$ & $20.97 \pm 0.01$ & $21.04 \pm 0.15$ & $21.44 \pm 0.01$ & $21.83 \pm 0.01$ & $22.48 \pm 0.02$ \\
\hline C18:1 11c & $1.1 \pm 0.02$ & $1.02 \pm 0.01$ & $1.03 \pm 0.01$ & $1.04 \pm 0.01$ & $1.06 \pm 0.01$ & $1.1 \pm 0.01$ \\
\hline $\mathrm{C} 18: 2 \mathrm{tt}$ & - & - & - & - & - & - \\
\hline $\mathrm{C} 18: 2 n-6$ & $54.96 \pm 0.36$ & $56.13 \pm 0.01$ & $55.62 \pm 0.42$ & $55.59 \pm 0.13$ & $54.91 \pm 0.1$ & $53.7 \pm 0.13$ \\
\hline $\mathrm{C} 18: 3 n-6$ & $0.44 \pm 0.01$ & $0.29 \pm 0.09$ & $0.3 \pm 0.01$ & $0.3 \pm 0$ & $0.3 \pm 0$ & $0.3 \pm 0$ \\
\hline $\mathrm{C} 18: 3 n-3$ & $6.25 \pm 0.06$ & $6.16 \pm 0.01$ & $6.01 \pm 0.05$ & $5.76 \pm 0.06$ & $5.49 \pm 0.05$ & $5.07 \pm 0.07$ \\
\hline C20:0 & $0.26 \pm 0.01$ & $0.24 \pm 0$ & $0.24 \pm 0$ & $0.24 \pm 0.01$ & $0.25 \pm 0.01$ & $0.27 \pm 0$ \\
\hline C20:1 & $0.15 \pm 0$ & $0.14 \pm 0.01$ & $0.14 \pm 0$ & $0.14 \pm 0$ & $0.14 \pm 0.01$ & $0.15 \pm 0.01$ \\
\hline C22:0 & $0.3 \pm 0.02$ & $0.28 \pm 0.1$ & $0.28 \pm 0.01$ & $0.29 \pm 0$ & $0.3 \pm 0.01$ & $0.31 \pm 0.01$ \\
\hline Other FAs & 2.39 & 0.68 & 1.16 & 0.72 & 0.72 & 0.91 \\
\hline$\Sigma$ SFA & 14.54 & 14.47 & 14.55 & 14.88 & 15.41 & 16.15 \\
\hline$\Sigma$ UFA & 83.07 & 84.85 & 84.29 & 84.4 & 83.88 & 82.94 \\
\hline$\Sigma \mathrm{TFA}$ & - & 0.05 & 0.05 & 0.04 & 0.05 & 0.08 \\
\hline
\end{tabular}

compared to the beginning (Tables 5, 9, and 10). After $60 \mathrm{~h}$ of processing heating at $210^{\circ} \mathrm{C}$, it has appeared as $\mathrm{C} 18: 2 \mathrm{t}$, $\mathrm{t}$ only in soybean and sunflower oils. SFA increased from $14.5 \%$ to $17.39 \%$, from $10.77 \%$ to $13.32 \%$, and from $14.49 \%$ to $16.82 \%$ in soybean, sunflower, and corn oils, respectively. However, UFA decreased by $84 \%$ to $80.72 \%$, from $88.38 \%$ to $84.37 \%$, and from $83.85 \%$ to $81.22 \%$ in soybean, sunflower, and corn oils, respectively. 
TABLE 5: Variation of fatty acids of soybean oil under $210^{\circ} \mathrm{C}$ heating temperature at different heating times.

\begin{tabular}{|c|c|c|c|c|c|c|}
\hline \multicolumn{7}{|c|}{ Heating time (hours)/fatty acids (\%) } \\
\hline & $0^{*}$ & 12 & 24 & 36 & 48 & 60 \\
\hline $\mathrm{C} 8: 0$ & - & $0.01 \pm 0.01$ & $0.02 \pm 0.01$ & $0.03 \pm 0.01$ & $0.11 \pm 0.01$ & $0.19 \pm 0$ \\
\hline $\mathrm{C} 12: 0$ & - & $0.03 \pm 0.01$ & $0.03 \pm 0$ & $0.03 \pm 0$ & $0.03 \pm 0$ & $0.03 \pm 0$ \\
\hline C14:0 & $0.05 \pm 0.01$ & $0.06 \pm 0.03$ & $0.07 \pm 0$ & $0.07 \pm 0$ & $0.08 \pm 0.01$ & $0.08 \pm 0$ \\
\hline $\mathrm{C} 16: 0$ & $10.33 \pm 0.12$ & $10.44 \pm 0.12$ & $10.52 \pm 0.01$ & $10.87 \pm 0.02$ & $11.43 \pm 0.02$ & $12.28 \pm 0$ \\
\hline $\mathrm{C} 16: 1$ & $0.06 \pm 0$ & $0.06 \pm 0.01$ & $0.06 \pm 0$ & $0.06 \pm 0$ & $0.07 \pm 0.01$ & $0.07 \pm 0$ \\
\hline $\mathrm{C} 17: 0$ & $0.07 \pm 0$ & $0.06 \pm 0.03$ & $0.08 \pm 0$ & $0.08 \pm 0$ & $0.09 \pm 0$ & $0.11 \pm 0$ \\
\hline C17:1 & $0.02 \pm 0.01$ & $0.03 \pm 0$ & $0.02 \pm 0.01$ & $0.03 \pm 0.01$ & $0.03 \pm 0$ & - \\
\hline C18:0 & $3.53 \pm 0.03$ & $3.46 \pm 0.05$ & $3.51 \pm 0.01$ & $3.61 \pm 0.01$ & $3.8 \pm 0.01$ & $4.08 \pm 0.01$ \\
\hline C18:1 9t & - & $0.02 \pm 0.01$ & $0.07 \pm 0.01$ & $0.07 \pm 0.01$ & $0.05 \pm 0.04$ & $0.17 \pm 0.01$ \\
\hline C18:1 9c & $21.23 \pm 0.14$ & $21.35 \pm 0.1$ & $21.59 \pm 0.02$ & $22.01 \pm 0.03$ & $22.73 \pm 0.02$ & $23.64 \pm 0.01$ \\
\hline $\mathrm{C} 18: 1 \mathrm{11c}$ & $1.1 \pm 0.02$ & $0.58 \pm 0.39$ & $1.03 \pm 0.01$ & $1.05 \pm 0$ & $1.1 \pm 0.01$ & $1.15 \pm 0$ \\
\hline $\mathrm{C} 18: 2 \mathrm{tt}$ & - & - & - & - & - & $0.07 \pm 0.02$ \\
\hline C18:2 $n-6$ & $54.96 \pm 0.36$ & $55.86 \pm 0.4$ & $55.6 \pm 0.1$ & $54.92 \pm 0.02$ & $53.55 \pm 0.07$ & $51.25 \pm 0.05$ \\
\hline $\mathrm{C} 18: 3 n-6$ & $0.44 \pm 0.01$ & $0.22 \pm 0.11$ & $0.37 \pm 0.01$ & $0.43 \pm 0.01$ & $0.48 \pm 0.01$ & $0.49 \pm 0$ \\
\hline $\mathrm{C} 18: 3 n-3$ & $6.25 \pm 0.06$ & $5.94 \pm 0.18$ & $5.35 \pm 0.05$ & $4.9 \pm 0.04$ & $4.33 \pm 0.05$ & $3.74 \pm 0.01$ \\
\hline C20:0 & $0.26 \pm 0.01$ & $0.2 \pm 0.09$ & $0.25 \pm 0.01$ & $0.25 \pm 0$ & $0.26 \pm 0.01$ & $0.28 \pm 0$ \\
\hline C20:1 & $0.15 \pm 0$ & $0.28 \pm 0.25$ & $0.14 \pm 0$ & $0.14 \pm 0$ & $0.15 \pm 0.01$ & $0.15 \pm 0$ \\
\hline C22:0 & $0.3 \pm 0.02$ & $0.25 \pm 0.09$ & $0.3 \pm 0.01$ & $0.3 \pm 0$ & $0.32 \pm 0$ & $0.34 \pm 0$ \\
\hline Other FAs & 2.39 & 1.15 & 0.98 & 1.16 & 1.41 & 1.89 \\
\hline$\Sigma$ SFA & 14.54 & 14.5 & 14.78 & 15.23 & 16.11 & 17.39 \\
\hline$\Sigma$ UFA & 83.07 & 84 & 84.24 & 83.6 & 82.47 & 80.72 \\
\hline$\Sigma \mathrm{TFA}$ & - & 0.02 & 0.07 & 0.07 & 0.05 & 0.24 \\
\hline
\end{tabular}

TABLE 6: Variation of fatty acids of soybean oil under $240^{\circ} \mathrm{C}$ heating temperature at different heating times.

\begin{tabular}{|c|c|c|c|c|c|c|}
\hline \multicolumn{7}{|c|}{ Heating time (hours)/fatty acids (\%) } \\
\hline & $0^{*}$ & 12 & 24 & 36 & 48 & 60 \\
\hline C8:0 & - & $0.05 \pm 0$ & $0.17 \pm 0$ & $0.36 \pm 0.01$ & $0.69 \pm 0.01$ & $1.24 \pm 0.02$ \\
\hline $\mathrm{C} 12: 0$ & - & $0.03 \pm 0$ & $0.04 \pm 0$ & $0.04 \pm 0.01$ & $0.06 \pm 0.01$ & $0.08 \pm 0$ \\
\hline C14:0 & $0.05 \pm 0.01$ & $0.07 \pm 0$ & $0.08 \pm 0$ & $0.1 \pm 0.01$ & $0.13 \pm 0.01$ & $0.17 \pm 0$ \\
\hline C16:0 & $10.33 \pm 0.12$ & $10.89 \pm 0.05$ & $12.31 \pm 0.01$ & $14.35 \pm 0.02$ & $17.46 \pm 0.18$ & $21.41 \pm 0.22$ \\
\hline C16:1 & $0.06 \pm 0$ & $0.06 \pm 0$ & $0.07 \pm 0$ & $0.08 \pm 0$ & $0.09 \pm 0$ & $0.09 \pm 0.01$ \\
\hline $\mathrm{C} 17: 0$ & $0.07 \pm 0$ & $0.08 \pm 0$ & $0.11 \pm 0$ & $0.17 \pm 0$ & $0.28 \pm 0.01$ & $0.58 \pm 0.01$ \\
\hline $\mathrm{C} 17: 1$ & $0.02 \pm 0.01$ & - & - & - & - & - \\
\hline C18:0 & $3.53 \pm 0.03$ & $3.62 \pm 0.01$ & $4.08 \pm 0.01$ & $4.77 \pm 0.01$ & $5.79 \pm 0.06$ & $7.06 \pm 0.08$ \\
\hline $\mathrm{C} 18: 19 \mathrm{t}$ & - & $0.07 \pm 0.02$ & $0.29 \pm 0.02$ & $0.55 \pm 0.03$ & $0.9 \pm 0.05$ & $1.38 \pm 0.2$ \\
\hline $\mathrm{C} 18: 19 \mathrm{c}$ & $21.23 \pm 0.14$ & $22.01 \pm 0.11$ & $23.88 \pm 0.02$ & $26.08 \pm 0.06$ & $29 \pm 0.29$ & $30.75 \pm 0.31$ \\
\hline C18:1 11c & $1.1 \pm 0.02$ & $1.06 \pm 0.01$ & $1.18 \pm 0.01$ & $1.32 \pm 0$ & $1.5 \pm 0.02$ & $1.63 \pm 0.02$ \\
\hline $\mathrm{C} 18: 2 \mathrm{tt}$ & - & $0.09 \pm 0.01$ & $0.22 \pm 0$ & $0.37 \pm 0.01$ & $0.48 \pm 0.01$ & $0.5 \pm 0.02$ \\
\hline $\mathrm{C} 18: 2 n-6$ & $54.96 \pm 0.36$ & $52.89 \pm 0.34$ & $48.68 \pm 0.03$ & $42.5 \pm 0.1$ & $35.21 \pm 0.34$ & $25.15 \pm 0.25$ \\
\hline $\mathrm{C} 18: 3 n-6$ & $0.44 \pm 0.01$ & $0.93 \pm 0.01$ & $1.02 \pm 0$ & $0.74 \pm 0.01$ & $0.44 \pm 0.01$ & $0.19 \pm 0.01$ \\
\hline $\mathrm{C} 18: 3 n-3$ & $6.25 \pm 0.06$ & $3.12 \pm 0.02$ & $1.41 \pm 0.02$ & $0.69 \pm 0.03$ & $0.34 \pm 0.04$ & - \\
\hline C20:0 & $0.26 \pm 0.01$ & $0.25 \pm 0.01$ & $0.29 \pm 0.01$ & $0.33 \pm 0.01$ & $0.42 \pm 0.01$ & $0.5 \pm 0.01$ \\
\hline C20:1 & $0.15 \pm 0$ & $0.16 \pm 0.01$ & $0.17 \pm 0.02$ & $0.17 \pm 0.01$ & $0.17 \pm 0.01$ & $0.16 \pm 0.01$ \\
\hline $\mathrm{C} 22: 0$ & $0.3 \pm 0.02$ & $0.31 \pm 0.01$ & $0.34 \pm 0$ & $0.45 \pm 0.08$ & $0.48 \pm 0.01$ & $0.56 \pm 0.01$ \\
\hline Other FAs & 2.39 & 4.33 & 5.66 & 6.92 & 6.56 & 8.55 \\
\hline$\Sigma$ SFA & 14.54 & 15.31 & 17.42 & 20.58 & 25.32 & 31.59 \\
\hline$\Sigma$ UFA & 83.07 & 80.36 & 76.92 & 72.5 & 68.12 & 59.86 \\
\hline$\Sigma \mathrm{TFA}$ & - & 0.16 & 0.51 & 0.92 & 1.38 & 1.88 \\
\hline
\end{tabular}

FA: fatty acid; SFA: saturated fatty acid; UFA: unsaturated fatty acid; TFA: trans-fatty acid. —: not detected; ${ }^{*}$ : fresh oil.

3.5. Effect of Treatment at $240^{\circ} \mathrm{C}$. The major saturated fatty acids (C16: 0 and C18: 0 ) were approximately 2 times higher after $60 \mathrm{~h}$ of treatment of all three oils. There was also a significant increase in minor SFA, especially of C8: 0 and C14: 0 in all oil samples. C12: 0 increased significantly in soybean, was present only in the last two stages in sunflower, and was absent in corn oil. The rate of C18: $19 \mathrm{c}$ increased significantly from $21.23 \%$ to $30.75 \%$, from $25.54 \%$ to $38.17 \%$, and from $30.70 \%$ to $44.01 \%$ at the end of the heat treatment process for soybean, sunflower, and corn oils, respectively (Tables 6, 11, and 12). Essential fatty acids such as C18: $2 n-6$ showed a decrease of more than $50 \%$ from the start of 
TABLE 7: Variation of fatty acids of sunflower oil under $180^{\circ} \mathrm{C}$ heating temperature at different heating times.

\begin{tabular}{|c|c|c|c|c|c|c|}
\hline \multicolumn{7}{|c|}{ Heating time (hours)/fatty acids (\%) } \\
\hline & $0^{*}$ & 12 & 24 & 36 & 48 & 60 \\
\hline C8:0 & - & - & - & $0.04 \pm 0.01$ & $0.08 \pm 0.01$ & $0.14 \pm 0.01$ \\
\hline C12:0 & - & - & - & - & - & - \\
\hline C14:0 & $0.05 \pm 0.01$ & $0.05 \pm 0.01$ & $0.05 \pm 0.01$ & $0.06 \pm 0.01$ & $0.06 \pm 0.01$ & $0.06 \pm 0.01$ \\
\hline $\mathrm{C} 16: 0$ & $6.29 \pm 0.18$ & $6.02 \pm 0.01$ & $5.99 \pm 0.03$ & $6.2 \pm 0.02$ & $6.4 \pm 0.12$ & $6.85 \pm 0.02$ \\
\hline C16:1 & $0.06 \pm 0.01$ & $0.07 \pm 0.01$ & $0.07 \pm 0.01$ & $0.07 \pm 0.01$ & $0.07 \pm 0.01$ & $0.08 \pm 0.01$ \\
\hline $\mathrm{C} 17: 0$ & $0.07 \pm 0.01$ & $0.03 \pm 0.01$ & $0.03 \pm 0.01$ & $0.03 \pm 0.01$ & $0.03 \pm 0.01$ & $0.04 \pm 0.01$ \\
\hline $\mathrm{C} 17: 1$ & - & - & - & - & - & - \\
\hline C18:0 & $3.44 \pm 0.19$ & $3.43 \pm 0.01$ & $3.41 \pm 0.02$ & $3.52 \pm 0.01$ & $3.49 \pm 0.17$ & $3.9 \pm 0.02$ \\
\hline $\mathrm{C} 18: 19 \mathrm{t}$ & - & $0.04 \pm 0.03$ & $0.06 \pm 0.01$ & $0.04 \pm 0.01$ & $0.04 \pm 0.02$ & $0.1 \pm 0.01$ \\
\hline $\mathrm{C} 18: 19 \mathrm{c}$ & $25.54 \pm 0.13$ & $26.74 \pm 0.02$ & $26.54 \pm 0.1$ & $27.24 \pm 0.01$ & $26.69 \pm 1.63$ & $29.17 \pm 0.02$ \\
\hline C18:1 11c & $1.29 \pm 0.33$ & $0.49 \pm 0.01$ & $0.47 \pm 0.01$ & $0.48 \pm 0.01$ & $0.51 \pm 0.04$ & $0.51 \pm 0.01$ \\
\hline $\mathrm{C} 18: 2 \mathrm{tt}$ & - & - & - & - & - & - \\
\hline $\mathrm{C} 18: 2 n-6$ & $55.53 \pm 0.95$ & $61.44 \pm 0.08$ & $59.15 \pm 0.23$ & $59.36 \pm 0.04$ & $59.39 \pm 2.14$ & $56.26 \pm 0.07$ \\
\hline $\mathrm{C} 18: 3 n-6$ & $0.43 \pm 0.03$ & - & - & - & - & - \\
\hline $\mathrm{C} 18: 3 n-3$ & $5.12 \pm 0.03$ & $0.15 \pm 0.02$ & $0.15 \pm 0.01$ & $0.14 \pm 0.01$ & $0.23 \pm 0.21$ & $0.1 \pm 0.02$ \\
\hline C20:0 & $0.25 \pm 0.03$ & $0.2 \pm 0.01$ & $0.19 \pm 0.01$ & $0.21 \pm 0.01$ & $0.21 \pm 0.02$ & $0.23 \pm 0.01$ \\
\hline C20:1 & $0.14 \pm 0.02$ & $0.09 \pm 0.01$ & $0.09 \pm 0.01$ & $0.1 \pm 0.01$ & $0.09 \pm 0.01$ & $0.1 \pm 0.01$ \\
\hline $\mathrm{C} 22: 0$ & $0.27 \pm 0.02$ & $0.54 \pm 0.01$ & $0.55 \pm 0.01$ & $0.58 \pm 0.01$ & $0.57 \pm 0.04$ & $0.62 \pm 0.01$ \\
\hline Other FAs & 1.52 & 0.72 & 3.25 & 1.93 & 2.13 & 1.84 \\
\hline$\Sigma$ SFA & 10.37 & 10.27 & 10.24 & 10.64 & 10.84 & 11.84 \\
\hline$\Sigma$ UFA & 88.11 & 89.01 & 86.51 & 87.43 & 87.03 & 86.32 \\
\hline$\Sigma$ TFA & - & 0.04 & 0.06 & 0.04 & 0.04 & 0.1 \\
\hline
\end{tabular}

TABLE 8: Variation of fatty acids of corn oil under $180^{\circ} \mathrm{C}$ heating temperature at different heating times.

\begin{tabular}{|c|c|c|c|c|c|c|}
\hline \multicolumn{7}{|c|}{ Heating time (hours)/fatty acids (\%) } \\
\hline & $0^{*}$ & 12 & 24 & 36 & 48 & 60 \\
\hline $\mathrm{C} 8: 0$ & - & - & - & - & - & $0.11 \pm 0.01$ \\
\hline $\mathrm{C} 12: 0$ & - & - & - & - & - & - \\
\hline C14:0 & $0.02 \pm 0.01$ & $0.02 \pm 0.01$ & $0.02 \pm 0.01$ & $0.02 \pm 0.01$ & $0.02 \pm 0.01$ & $0.03 \pm 0.01$ \\
\hline C16:0 & $10.28 \pm 0.11$ & $12.29 \pm 0.01$ & $12.23 \pm 0.07$ & $12.46 \pm 0.06$ & $12.67 \pm 0.07$ & $13.75 \pm 0.14$ \\
\hline $\mathrm{C} 16: 1 \mathrm{t}$ & $0.03 \pm 0$ & $0.03 \pm 0.01$ & $0.03 \pm 0.01$ & $0.03 \pm 0.01$ & $0.03 \pm 0.01$ & $0.03 \pm 0.01$ \\
\hline C16:1 c & $0.08 \pm 0$ & $0.1 \pm 0.01$ & $0.1 \pm 0.01$ & $0.1 \pm 0.01$ & $0.1 \pm 0.01$ & $0.11 \pm 0.01$ \\
\hline $\mathrm{C} 17: 0$ & $0.05 \pm 0.01$ & $0.06 \pm 0.01$ & $0.06 \pm 0.01$ & $0.06 \pm 0.01$ & $0.07 \pm 0.01$ & $0.08 \pm 0.01$ \\
\hline $\mathrm{C} 17: 1$ & $0.02 \pm 0.01$ & $0.03 \pm 0.01$ & - & $0.03 \pm 0.01$ & - & $0.05 \pm 0.04$ \\
\hline C18:0 & $1.49 \pm 0.02$ & $1.79 \pm 0.02$ & $1.79 \pm 0.01$ & $1.82 \pm 0.01$ & $1.86 \pm 0.01$ & $2.01 \pm 0.02$ \\
\hline C18:1 9t & $0.05 \pm 0.01$ & $0.06 \pm 0.03$ & $0.05 \pm 0.01$ & $0.06 \pm 0.02$ & $0.08 \pm 0.01$ & $0.1 \pm 0.05$ \\
\hline $\mathrm{C} 18: 19 \mathrm{c}$ & $30.7 \pm 0.27$ & $34.64 \pm 0.08$ & $34.53 \pm 0.32$ & $34.98 \pm 0.24$ & $35.37 \pm 0.25$ & $37 \pm 0.36$ \\
\hline C18:1 11c & $0.47 \pm 0.01$ & $0.47 \pm 0.01$ & $0.47 \pm 0.01$ & $0.47 \pm 0.01$ & $0.48 \pm 0.01$ & $0.5 \pm 0.01$ \\
\hline $\mathrm{C} 18: 2 \mathrm{tt}$ & - & - & - & - & - & - \\
\hline $\mathrm{C} 18: 2 n-6$ & $53.66 \pm 0.13$ & $48.31 \pm 0.14$ & $47.71 \pm 0.46$ & $47.53 \pm 0.35$ & $46.66 \pm 0.33$ & $43.51 \pm 0.45$ \\
\hline $\mathrm{C} 18: 3 n-6$ & $0.05 \pm 0.01$ & - & - & - & - & - \\
\hline $\mathrm{C} 18: 3 n-3$ & $0.72 \pm 0.01$ & $0.63 \pm 0.06$ & $0.6 \pm 0.05$ & $0.58 \pm 0.04$ & $0.57 \pm 0.05$ & $0.47 \pm 0.05$ \\
\hline $\mathrm{C} 20: 0$ & $0.38 \pm 0.01$ & $0.39 \pm 0.01$ & $0.4 \pm 0.01$ & $0.4 \pm 0.01$ & $0.41 \pm 0.01$ & $0.44 \pm 0.02$ \\
\hline C20:1 & $0.2 \pm 0.01$ & $0.18 \pm 0.01$ & $0.18 \pm 0.01$ & $0.18 \pm 0.01$ & $0.19 \pm 0.01$ & $0.2 \pm 0.01$ \\
\hline $\mathrm{C} 22: 0$ & $0.09 \pm 0.01$ & - & - & - & - & - \\
\hline Other FAs & 1.71 & 1 & 1.83 & 1.28 & 1.49 & 1.62 \\
\hline$\Sigma$ SFA & 12.31 & 14.55 & 14.50 & 13.76 & 14.76 & 16.42 \\
\hline$\Sigma U F A$ & 85.98 & 84.45 & 83.68 & 84.69 & 83.96 & 81.97 \\
\hline$\Sigma \mathrm{TFA}$ & 0.08 & 0.09 & 0.08 & 0.09 & 0.11 & 0.13 \\
\hline
\end{tabular}

FA: fatty acid; SFA: saturated fatty acid; UFA: unsaturated fatty acid; TFA: trans-fatty acid. —: not detected; * fresh oil.

the treatment at $240^{\circ} \mathrm{C}$ for all three oils. C18: $3 n-3$ was missing in time $t=24 \mathrm{~h}$ for sunflower, from $t=36 \mathrm{~h}$ for corn, and at $t=60 \mathrm{~h}$ for soybean. C18: $3 n-6$ decreased during the different stages of the heating process in soybean and corn, while it was absent in sunflower.
The rate of trans-fatty acids (18: $19 \mathrm{t}$; C18: $2 \mathrm{tt})$ increased in proportion to the heating time for all three oil categories (Figure 4) due to the oxidative deterioration of these oils. The increase in TFA was associated with a decrease in UFA, suggesting that PUFA could be the TFA precursors by 
TABLE 9: Variation of fatty acids of sunflower oil under $210^{\circ} \mathrm{C}$ heating temperature at different heating times.

\begin{tabular}{|c|c|c|c|c|c|c|}
\hline \multicolumn{7}{|c|}{ Heating time (hours)/fatty acids (\%) } \\
\hline & $0^{*}$ & 12 & 24 & 36 & 48 & 60 \\
\hline $\mathrm{C} 8: 0$ & - & $0.03 \pm 0$ & $0.04 \pm 0.01$ & $0.07 \pm 0.01$ & $0.14 \pm 0.01$ & $0.29 \pm 0.01$ \\
\hline $\mathrm{C} 12: 0$ & - & - & - & - & - & - \\
\hline $\mathrm{C} 14: 0$ & $0.05 \pm 0.01$ & $0.06 \pm 0$ & $0.05 \pm 0.01$ & $0.06 \pm 0.01$ & $0.06 \pm 0.01$ & $0.07 \pm 0.01$ \\
\hline $\mathrm{C} 16: 0$ & $6.29 \pm 0.18$ & $6.33 \pm 0$ & $6.25 \pm 0.01$ & $6.47 \pm 0.03$ & $6.84 \pm 0.02$ & $7.63 \pm 0.02$ \\
\hline C16:1 & $0.06 \pm 0.01$ & $0.07 \pm 0$ & $0.07 \pm 0.01$ & $0.07 \pm 0.01$ & $0.07 \pm 0.01$ & $0.08 \pm 0.01$ \\
\hline $\mathrm{C} 17: 0$ & $0.07 \pm 0.01$ & $0.03 \pm 0$ & $0.03 \pm 0.01$ & $0.04 \pm 0.01$ & $0.05 \pm 0.01$ & $0.07 \pm 0.01$ \\
\hline $\mathrm{C} 17: 1$ & - & - & - & - & - & - \\
\hline C18:0 & $3.44 \pm 0.19$ & $3.54 \pm 0$ & $3.55 \pm 0.01$ & $3.67 \pm 0.02$ & $3.88 \pm 0.02$ & $4.3 \pm 0.01$ \\
\hline $\mathrm{C} 18: 19 \mathrm{t}$ & - & $0.06 \pm 0$ & $0.07 \pm 0.02$ & $0.06 \pm 0.01$ & $0.09 \pm 0.03$ & $0.25 \pm 0.02$ \\
\hline $\mathrm{C} 18: 19 \mathrm{c}$ & $25.54 \pm 0.13$ & $27.23 \pm 0$ & $27.49 \pm 0.03$ & $28.11 \pm 0.02$ & $29.09 \pm 0.03$ & $30.84 \pm 0.14$ \\
\hline C18:1 11c & $1.29 \pm 0.33$ & $0.46 \pm 0$ & $0.48 \pm 0.01$ & $0.5 \pm 0.01$ & $0.53 \pm 0.01$ & $0.57 \pm 0.01$ \\
\hline $\mathrm{C} 18: 2 \mathrm{tt}$ & - & - & - & - & - & $0.1 \pm 0.01$ \\
\hline $\mathrm{C} 18: 2 n-6$ & $55.53 \pm 0.95$ & $60.35 \pm 0$ & $59.84 \pm 0.05$ & $58.65 \pm 0.1$ & $56.61 \pm 0.15$ & $52.36 \pm 0.29$ \\
\hline $\mathrm{C} 18: 3 n-6$ & $0.43 \pm 0.03$ & - & - & - & - & - \\
\hline $\mathrm{C} 18: 3 n-3$ & $5.12 \pm 0.03$ & $0.11 \pm 0$ & $0.12 \pm 0.01$ & $0.1 \pm 0.02$ & $0.09 \pm 0.02$ & $0.07 \pm 0.02$ \\
\hline C20:0 & $0.25 \pm 0.03$ & $0.21 \pm 0$ & $0.2 \pm 0.01$ & $0.21 \pm 0.01$ & $0.23 \pm 0.01$ & $0.25 \pm 0.01$ \\
\hline C20:1 & $0.14 \pm 0.02$ & $0.1 \pm 0$ & $0.1 \pm 0.01$ & $0.1 \pm 0.01$ & $0.1 \pm 0.01$ & $0.11 \pm 0.01$ \\
\hline $\mathrm{C} 22: 0$ & $0.27 \pm 0.02$ & $0.57 \pm 0$ & $0.58 \pm 0.01$ & $0.6 \pm 0.01$ & $0.62 \pm 0.03$ & $0.7 \pm 0.01$ \\
\hline Other FAs & 1.52 & 0.85 & 1.12 & 1.29 & 1.54 & 2.31 \\
\hline$\Sigma$ SFA & 10.37 & 10.77 & 10.71 & 11.12 & 11.83 & 13.32 \\
\hline$\Sigma$ UFA & 88.11 & 88.38 & 88.17 & 87.59 & 86.63 & 84.37 \\
\hline$\Sigma \mathrm{TFA}$ & - & 0.06 & 0.07 & 0.06 & 0.09 & 0.35 \\
\hline
\end{tabular}

TABLE 10: Variation of fatty acids of corn oil under $210^{\circ} \mathrm{C}$ heating temperature at different heating times.

\begin{tabular}{|c|c|c|c|c|c|c|}
\hline \multicolumn{7}{|c|}{ Heating time (hours)/fatty acids (\%) } \\
\hline & $0^{*}$ & 12 & 24 & 36 & 48 & 60 \\
\hline $\mathrm{C} 8: 0$ & - & - & - & - & - & $0.14 \pm 0.01$ \\
\hline $\mathrm{C} 12: 0$ & - & - & - & - & - & - \\
\hline C14:0 & $0.02 \pm 0.01$ & $0.02 \pm 0.01$ & $0.02 \pm 0.01$ & $0.02 \pm 0.01$ & $0.1 \pm 0.01$ & $0.03 \pm 0.01$ \\
\hline $\mathrm{C} 16: 0$ & $10.28 \pm 0.11$ & $12.22 \pm 0.08$ & $12.45 \pm 0.03$ & $12.62 \pm 0.12$ & $13.45 \pm 0.58$ & $14.05 \pm 0.07$ \\
\hline C16:1t & $0.03 \pm 0$ & $0.03 \pm 0.01$ & $0.03 \pm 0.01$ & $0.03 \pm 0.01$ & $0.03 \pm 0.01$ & $0.06 \pm 0.05$ \\
\hline $\mathrm{C} 16: 1 \mathrm{c}$ & $0.08 \pm 0$ & $0.1 \pm 0.01$ & $0.1 \pm 0.01$ & $0.1 \pm 0.01$ & $0.1 \pm 0.01$ & $0.11 \pm 0.01$ \\
\hline $\mathrm{C} 17: 0$ & $0.05 \pm 0.01$ & $0.06 \pm 0.01$ & $0.06 \pm 0.01$ & $0.06 \pm 0.01$ & $0.08 \pm 0.01$ & $0.08 \pm 0.01$ \\
\hline $\mathrm{C} 17: 1$ & $0.02 \pm 0.01$ & - & - & - & - & - \\
\hline C18:0 & $1.49 \pm 0.02$ & $1.79 \pm 0.01$ & $1.82 \pm 0.01$ & $1.85 \pm 0.03$ & $1.97 \pm 0.09$ & $2.06 \pm 0.01$ \\
\hline $\mathrm{C} 18: 19 \mathrm{t}$ & $0.05 \pm 0.01$ & $0.06 \pm 0.01$ & $0.05 \pm 0.01$ & $0.09 \pm 0.01$ & $0.19 \pm 0.03$ & $0.26 \pm 0.05$ \\
\hline C18:1 9c & $30.7 \pm 0.27$ & $34.57 \pm 0.24$ & $34.98 \pm 0.16$ & $35.22 \pm 0.09$ & $36.49 \pm 1$ & $37.34 \pm 0.26$ \\
\hline C18:1 11c & $0.47 \pm 0.01$ & $0.47 \pm 0.01$ & $0.47 \pm 0.01$ & $0.47 \pm 0.01$ & $0.51 \pm 0.04$ & $0.53 \pm 0.02$ \\
\hline $\mathrm{C} 18: 2 \mathrm{tt}$ & - & - & - & - & - & - \\
\hline $\mathrm{C} 18: 2 n-6$ & $53.66 \pm 0.13$ & $47.85 \pm 0.35$ & $47.39 \pm 0.24$ & $46.74 \pm 0.9$ & $44.05 \pm 1.31$ & $42.27 \pm 0.31$ \\
\hline $\mathrm{C} 18: 3 n-6$ & $0.05 \pm 0.01$ & - & - & $0.06 \pm 0.01$ & $0.06 \pm 0.01$ & $0.05 \pm 0.01$ \\
\hline $\mathrm{C} 18: 3 n-3$ & $0.72 \pm 0.01$ & $0.59 \pm 0.04$ & $0.2 \pm 0.27$ & $0.53 \pm 0.02$ & $0.44 \pm 0.1$ & $0.4 \pm 0.06$ \\
\hline C20:0 & $0.38 \pm 0.01$ & $0.4 \pm 0.02$ & $0.51 \pm 0.09$ & $0.41 \pm 0.01$ & $0.43 \pm 0.03$ & $0.45 \pm 0.02$ \\
\hline C20:1 & $0.2 \pm 0.01$ & $0.18 \pm 0.01$ & $0.33 \pm 0.13$ & $0.19 \pm 0.01$ & $0.19 \pm 0.01$ & $0.2 \pm 0.01$ \\
\hline C22:0 & $0.09 \pm 0.01$ & - & - & - & - & - \\
\hline Other FAs & 1.71 & 1.65 & 1.57 & 1.61 & 1.92 & 1.95 \\
\hline$\Sigma$ SFA & 12.31 & 14.49 & 14.87 & 14.96 & 16.05 & 16.82 \\
\hline$\Sigma U F A$ & 85.98 & 83.85 & 83.56 & 83.43 & 82.03 & 81.22 \\
\hline$\Sigma \mathrm{TFA}$ & 0.08 & 0.09 & 0.08 & 0.12 & 0.22 & 0.32 \\
\hline
\end{tabular}

FA: fatty acid; SFA: saturated fatty acid; UFA: unsaturated fatty acid; TFA: trans-fatty acid. —: not detected; * fresh oil.

chemical transformation [40]. These results are in agreement with those found by $[41,42]$, who suggest that the isomers and trans-fatty acids produced during the heating treatment depend mainly on the temperature reached, but also on the application processing time.
The increase in SFA leads to a decrease in UFA as the duration of treatment increases. These results are in line with those described by [43], who mentioned that the unsaturated fatty acids are more easily transformed. The intensity of the change in lipids depends on their 
TABLE 11: Variation of fatty acids of sunflower oil under $240^{\circ} \mathrm{C}$ heating temperature at different heating times.

\begin{tabular}{|c|c|c|c|c|c|c|}
\hline \multicolumn{7}{|c|}{ Heating time (hours)/fatty acids (\%) } \\
\hline & $0^{*}$ & 12 & 24 & 36 & 48 & 60 \\
\hline C8:0 & - & $0.04 \pm 0.01$ & $0.12 \pm 0.01$ & $0.28 \pm 0.02$ & $0.56 \pm 0.03$ & $1.21 \pm 0.06$ \\
\hline $\mathrm{C} 12: 0$ & - & - & - & - & $0.03 \pm 0.01$ & $0.05 \pm 0.01$ \\
\hline C14:0 & $0.05 \pm 0.01$ & $0.06 \pm 0.01$ & $0.06 \pm 0.01$ & $0.07 \pm 0.01$ & $0.09 \pm 0.01$ & $0.13 \pm 0.01$ \\
\hline $\mathrm{C} 16: 0$ & $6.29 \pm 0.18$ & $6.32 \pm 0.02$ & $6.76 \pm 0.02$ & $7.68 \pm 0.03$ & $9.09 \pm 0.01$ & $11.69 \pm 0.04$ \\
\hline C16:1 & $0.06 \pm 0.01$ & $0.07 \pm 0.01$ & $0.07 \pm 0.01$ & $0.08 \pm 0.01$ & $0.09 \pm 0.01$ & $0.11 \pm 0.01$ \\
\hline $\mathrm{C} 17: 0$ & $0.07 \pm 0.01$ & $0.03 \pm 0.01$ & $0.05 \pm 0.01$ & $0.07 \pm 0.01$ & $0.13 \pm 0.01$ & $0.29 \pm 0.01$ \\
\hline C17:1 & - & - & - & - & - & - \\
\hline C18:0 & $3.44 \pm 0.19$ & $3.58 \pm 0.02$ & $3.82 \pm 0.02$ & $4.36 \pm 0.01$ & $5.13 \pm 0.01$ & $6.6 \pm 0.02$ \\
\hline $\mathrm{C} 18: 19 \mathrm{t}$ & - & $0.08 \pm 0.02$ & $0.23 \pm 0.03$ & $0.46 \pm 0.03$ & $0.77 \pm 0.04$ & $1.25 \pm 0.03$ \\
\hline C18:1 9c & $25.54 \pm 0.13$ & $27.67 \pm 0.08$ & $28.7 \pm 0.02$ & $31.2 \pm 0.02$ & $33.9 \pm 0.11$ & $38.17 \pm 0.01$ \\
\hline $\mathrm{C} 18: 1 \mathrm{11c}$ & $1.29 \pm 0.33$ & $0.5 \pm 0.01$ & $0.53 \pm 0.01$ & $0.61 \pm 0.02$ & $0.68 \pm 0.01$ & $0.79 \pm 0.03$ \\
\hline $\mathrm{C} 18: 2 \mathrm{tt}$ & - & $0.08 \pm 0.01$ & $0.2 \pm 0.01$ & $0.35 \pm 0.01$ & $0.45 \pm 0.01$ & $0.49 \pm 0.01$ \\
\hline $\mathrm{C} 18: 2 n-6$ & $55.53 \pm 0.95$ & $58.04 \pm 0.3$ & $52.05 \pm 0.06$ & $46.19 \pm 0.11$ & $38.35 \pm 0.16$ & $27.46 \pm 0.01$ \\
\hline $\mathrm{C} 18: 3 n-6$ & $0.43 \pm 0.03$ & - & - & - & - & - \\
\hline $\mathrm{C} 18: 3 n-3$ & $5.12 \pm 0.03$ & $0.06 \pm 0.02$ & - & - & - & - \\
\hline C20:0 & $0.25 \pm 0.03$ & $0.21 \pm 0.01$ & $0.23 \pm 0.01$ & $0.26 \pm 0.01$ & $0.31 \pm 0.01$ & $0.41 \pm 0.01$ \\
\hline C20:1 & $0.14 \pm 0.02$ & $0.1 \pm 0.01$ & $0.11 \pm 0.01$ & $0.1 \pm 0.01$ & $0.11 \pm 0.01$ & - \\
\hline C22:0 & $0.27 \pm 0.02$ & $0.59 \pm 0.01$ & $0.62 \pm 0.01$ & $0.71 \pm 0.02$ & $0.83 \pm 0.02$ & $1.06 \pm 0.03$ \\
\hline Other FAs & 1.52 & 2.58 & 6.45 & 7.58 & 9.47 & 10.29 \\
\hline$\Sigma$ SFA & 10.37 & 10.83 & 11.66 & 13.43 & 16.17 & 21.44 \\
\hline$\Sigma$ UFA & 88.11 & 86.59 & 81.89 & 78.99 & 74.36 & 68.27 \\
\hline$\Sigma \mathrm{TFA}$ & - & 0.16 & 0.43 & 0.81 & 1.22 & 1.74 \\
\hline
\end{tabular}

FA: fatty acid; SFA: saturated fatty acid; UFA: unsaturated fatty acid; TFA: trans-fatty acid. —: not detected; * fresh oil.

TABle 12: Variation of fatty acids of corn oil under $240^{\circ} \mathrm{C}$ heating temperature at different heating times.

\begin{tabular}{|c|c|c|c|c|c|c|}
\hline \multicolumn{7}{|c|}{ Heating time (hours)/fatty acids (\%) } \\
\hline & $0^{*}$ & 12 & 24 & 36 & 48 & 60 \\
\hline C8:0 & - & - & $0.08 \pm 0.01$ & $0.19 \pm 0.01$ & $0.37 \pm 0.01$ & $0.78 \pm 0.05$ \\
\hline $\mathrm{C} 12: 0$ & - & - & - & - & - & - \\
\hline C14:0 & $0.02 \pm 0.03$ & $0.02 \pm 0.01$ & $0.03 \pm 0.01$ & $0.03 \pm 0.01$ & $0.03 \pm 0.01$ & $0.05 \pm 0.01$ \\
\hline $\mathrm{C} 16: 0$ & $10.28 \pm 0.11$ & $12.72 \pm 0.07$ & $13.61 \pm 0.05$ & $15.07 \pm 0.01$ & $17.15 \pm 0.3$ & $20.82 \pm 0.39$ \\
\hline C16:1t & $0.03 \pm 0$ & $0.03 \pm 0.01$ & $0.03 \pm 0.01$ & $0.03 \pm 0.01$ & $0.04 \pm 0.01$ & $0.04 \pm 0.01$ \\
\hline $\mathrm{C} 16: 1 \mathrm{c}$ & $0.08 \pm 0$ & $0.1 \pm 0.01$ & $0.11 \pm 0.01$ & $0.11 \pm 0.01$ & $0.12 \pm 0.01$ & $0.13 \pm 0.01$ \\
\hline $\mathrm{C} 17: 0$ & $0.05 \pm 0.01$ & $0.06 \pm 0.01$ & $0.07 \pm 0.01$ & $0.1 \pm 0.01$ & $0.17 \pm 0.01$ & $0.32 \pm 0.02$ \\
\hline $\mathrm{C} 17: 1$ & $0.02 \pm 0.01$ & - & - & - & - & - \\
\hline C18:0 & $1.49 \pm 0.02$ & $1.83 \pm 0.01$ & $1.96 \pm 0.01$ & $2.17 \pm 0.01$ & $2.5 \pm 0.05$ & $3.01 \pm 0.06$ \\
\hline $\mathrm{C} 18: 19 \mathrm{t}$ & $0.05 \pm 0.01$ & $0.11 \pm 0.05$ & $0.28 \pm 0.01$ & $0.55 \pm 0.03$ & $0.87 \pm 0.02$ & $1.41 \pm 0.06$ \\
\hline $\mathrm{C} 18: 19 \mathrm{c}$ & $30.7 \pm 0.27$ & $35.75 \pm 0.31$ & $37.35 \pm 0.16$ & $39.53 \pm 0.11$ & $41.62 \pm 0.76$ & $44.01 \pm 0.86$ \\
\hline C18:1 11c & $0.47 \pm 0.01$ & $0.47 \pm 0.01$ & $0.52 \pm 0.01$ & $0.58 \pm 0.01$ & $0.63 \pm 0.02$ & $0.7 \pm 0.03$ \\
\hline $\mathrm{C} 18: 2 \mathrm{tt}$ & - & $0.06 \pm 0.01$ & $0.15 \pm 0.01$ & $0.25 \pm 0.01$ & $0.33 \pm 0.01$ & $0.35 \pm 0.01$ \\
\hline $\mathrm{C} 18: 2 n-6$ & $53.66 \pm 0.13$ & $45.18 \pm 0.38$ & $41.29 \pm 0.17$ & $36.06 \pm 0.08$ & $29.44 \pm 0.53$ & $20.36 \pm 0.34$ \\
\hline $\mathrm{C} 18: 3 n-6$ & $0.05 \pm 0.01$ & $0.1 \pm 0.01$ & $0.11 \pm 0.01$ & $0.08 \pm 0.01$ & - & - \\
\hline $\mathrm{C} 18: 3 n-3$ & $0.72 \pm 0.01$ & $0.34 \pm 0.03$ & $0.16 \pm 0.02$ & - & - & - \\
\hline C20:0 & $0.38 \pm 0.01$ & $0.42 \pm 0.01$ & $0.44 \pm 0.01$ & $0.49 \pm 0.01$ & $0.55 \pm 0.02$ & $0.68 \pm 0.03$ \\
\hline C20:1 & $0.2 \pm 0.01$ & $0.19 \pm 0.01$ & $0.21 \pm 0.01$ & $0.22 \pm 0.02$ & $0.22 \pm 0.01$ & $0.23 \pm 0.01$ \\
\hline C22:0 & $0.09 \pm 0.01$ & - & - & - & - & - \\
\hline Other FAs & 1.71 & 2.61 & 3.60 & 4.53 & 5.98 & 7.11 \\
\hline$\Sigma$ SFA & 12.31 & 15.05 & 16.18 & 14.54 & 20.76 & 25.67 \\
\hline$\Sigma$ UFA & 85.98 & 82.34 & 80.21 & 82.43 & 73.26 & 67.23 \\
\hline$\Sigma \mathrm{TFA}$ & 0.08 & 0.2 & 0.45 & 0.83 & 1.24 & 1.8 \\
\hline
\end{tabular}

FA: fatty acid; SFA: saturated fatty acid; UFA: unsaturated fatty acid; TFA: trans-fatty acid. —: not detected; ${ }^{*}$ : fresh oil. Each value represents the mean \pm standard deviation $(\mathrm{SD})$ of three determinations. *

physicochemical properties, in particular the degree of unsaturation of fatty acids.

The $n-3$ PUFA are sensitive to oxidative and thermal degradation. For example, in contact with oxygen, $n-3$
PUFA degraded into polar compounds, undesirable in a healthy diet [30]. However, during prolonged heat treatment, $n-3$ PUFA deteriorate into trans-fatty acids, polymers, and cyclic fatty acid monomers (MCFA), products 


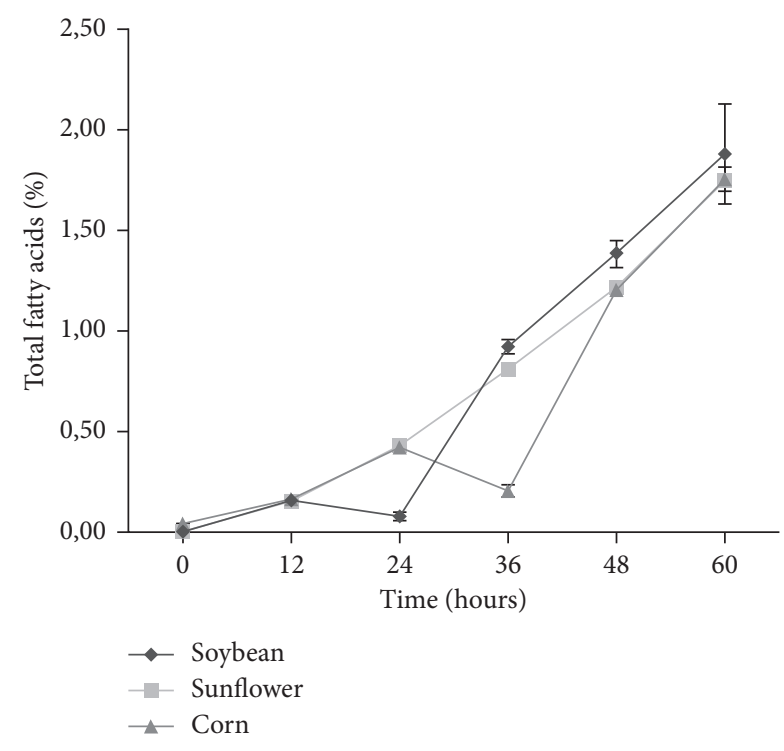

FIGURE 4: Occurrence of trans-fatty acids during the heating process from 0 hours (fresh oil) to 60 hours at $240^{\circ} \mathrm{C}$ of soybean, sunflower, and corn oils.

whose harmfulness is either known or questioned [24, 44-47]. Compared to polyunsaturated fatty acids, oleic acid was more stable to oxidation at high temperatures, which prevailed during frying and cooking.

Oils rich in linoleic and linolenic acids are less heattolerant. As the oil was heated, the amount of new chemical compounds was high for all three oils, which was consistent with the results reported by [48]. These authors suggested the presence of new chemical species, which are found in small amounts in peanut and palm oils and higher for rapeseed, soybean, and sunflower.

The number of new chemical species was proportional to the temperature increase. If the temperature exceeded $200^{\circ} \mathrm{C}$ or if the heating was very long at $180^{\circ} \mathrm{C}-190^{\circ} \mathrm{C}$, cyclic monomers appeared [49].

3.6. The Temperature Effect on Oleic Acid (C18: 1) Rate. As shown in Tables 1-4, 7, and 8, C18: 1 remained relatively stable during heat treatment temperatures at 150 and $180^{\circ} \mathrm{C}$. For corn oil, C18: 1 ranged from $34.63 \%$ after 12 hours to $34.25 \%$ after 60 hours of treatment at $150^{\circ} \mathrm{C}$ and from $34.64 \%$ after 12 hours to $37 \%$ after 60 hours of treatment at $180^{\circ} \mathrm{C}$.

However, there was a significant variation in the C18: 1 rate of corn oil at $210^{\circ} \mathrm{C}$ and more particularly at $240^{\circ} \mathrm{C}$ (Tables 10 and 12). In fact, this content gradually increased from $35.75 \%$ after 12 hours to $44.01 \%$ after 60 hours. This suggested that C18: 1 content began to vary significantly from $210^{\circ} \mathrm{C}$ and depending on the duration of treatment. This behavior was the same for the three oils.

3.7. The Temperature Effect on Polyunsaturated Fatty Acids (PUFA) Rate. First, it should be noted that in fresh oils, C18: 2 is the highest fatty acid among total fatty acids and accounted for $55.53 \%, 54.96 \%$, and $53.66 \%$, respectively, in sunflower, soybean, and corn oils. The PUFA content w6 (C18: $2 n-6)$ and w3 (C18: $3 n-3)$ are slightly affected by heating temperatures at 150 and $180^{\circ} \mathrm{C}$ and at different treatment times $(12,24,36,48$, and 60 hours).

However, the results showed a significant decrease in these fatty acid levels from $210^{\circ} \mathrm{C}$, and they generally dropped below $50 \%$ at the end of treatment $(60 \mathrm{~h})$ at $240^{\circ} \mathrm{C}$. At this temperature, the C18: $2 n-6$ and C18: $3 n-3$ content were more sensitive to the thermal effect than other fatty acids, which is in agreement with the results found by [50].

Furthermore, the literature mainly describes results regarding the effect of heat treatment on linoleic acid (C18: $2 n-6)$ and $\alpha$-linolenic acid (C18: $3 n-3)$ [42, 51]. Indeed, these authors showed that $\alpha$-linolenic acid was more sensitive to isomerization than linoleic acid.

The intensity of changes in lipids depends on their physicochemical properties; in particular, the degree of unsaturation makes it easier for unsaturated fatty acids to transform under the thermal effect $[41,43]$. Our results show a slight decrease in the PUFAs W6 (C18: 2n-6) and w3 (C18: $2 n-3)$ at $150^{\circ} \mathrm{C}$. This decrease becomes more important at $180^{\circ} \mathrm{C}$. However, subjected to the higher temperatures, $210^{\circ} \mathrm{C}$ and $240^{\circ} \mathrm{C}$, there was a more advanced alteration of PUFA associated with an increase in saturated fatty acids (SFA) in all oil samples (Figure 5).

Thermal treatment resulted in a decrease in the ratio of polyunsaturated fatty acids to monounsaturated fatty acids (PUFAs/MUFAs) (Figure 6), the latter decreases with the increase in temperature. The determination of this ratio could be likely an indicator of overheating or excessive oil use.

Therefore, the transformation of PUFA becomes important when samples have been subjected to high temperatures and for a long time. The two parameters (processing time and high temperature) were an amplifying factor in the degradation of the treated oils.

The stability of edible oils is not only determined by the fatty acid composition but is also affected by the presence of tocopherols and phytosterols. If tocopherols are degraded in the presence of molecular oxygen and produce oxidized products, this causes a loss of their antioxidant activity [52].

Despite the presence of almost the same PUFA composition of soybean and sunflower, sunflower oil is more oxidized than soybean oil. This could be explained by the richness of soybean oil in antioxidants compared to sunflower. In fact, soybean oil was considered a rich source of tocopherols (vitamin E) as reported by [53]. The tocopherol content of various soybean seed oil varieties ranged from 90.74 to $1011.87 \mathrm{mg} / \mathrm{kg}$; however, the tocopherol content in sunflower seed ranged from 116.2 to $122.7 \mathrm{mg} / \mathrm{kg}$ [14].

The decrease in the essential fatty acid content could be accounted for by the sensitivity of the double bonds to the increase in temperature. However, the presence of a significant amount of antioxidant compounds could protect the oil against oxidation when the oil is heated for an extended period.

The heat treatment involves a change in the fatty acid composition of the oil. This change was due to the 


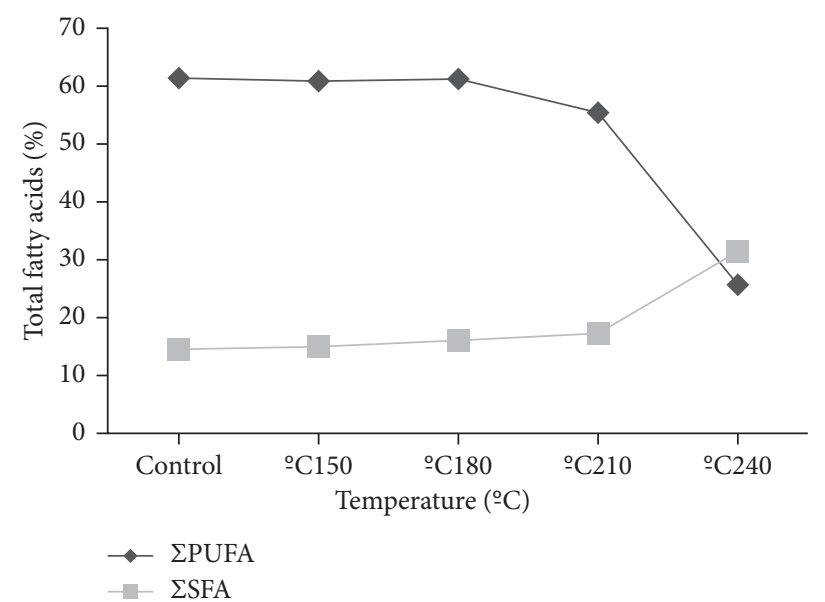

(a)

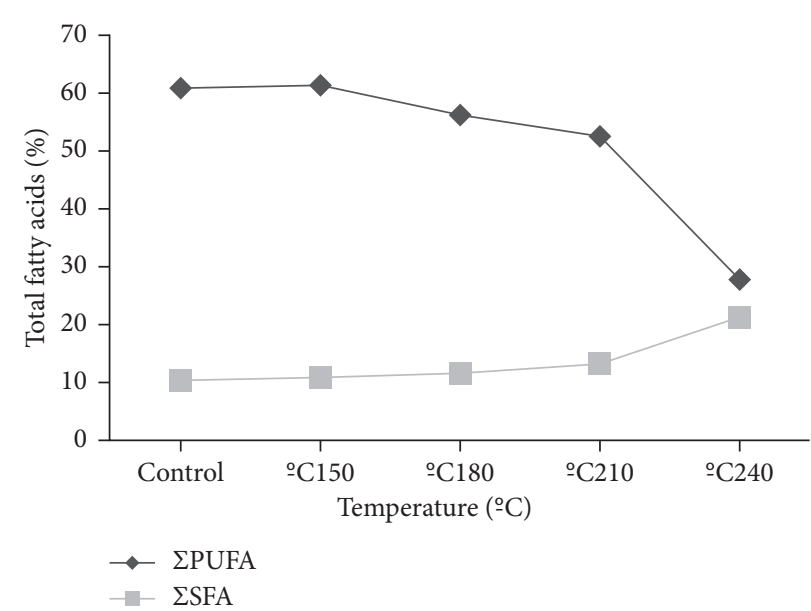

(b)

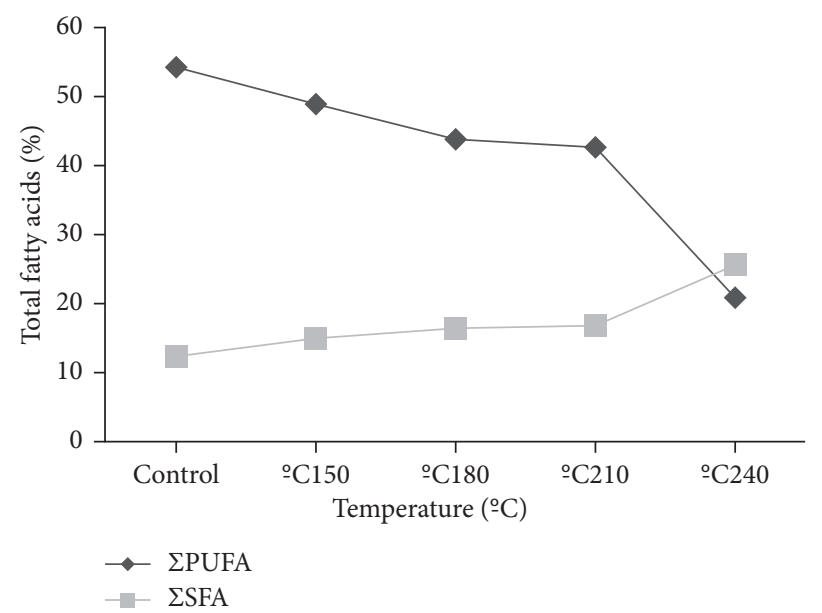

(c)

FIGURE 5: Change in the sum of polyunsaturated fatty acids ( $\Sigma$ PUFAs) and saturated fatty acids ( $\Sigma$ SFAs) during heating of soybean (a), sunflower (b), and corn oils (c) after 60 hours.

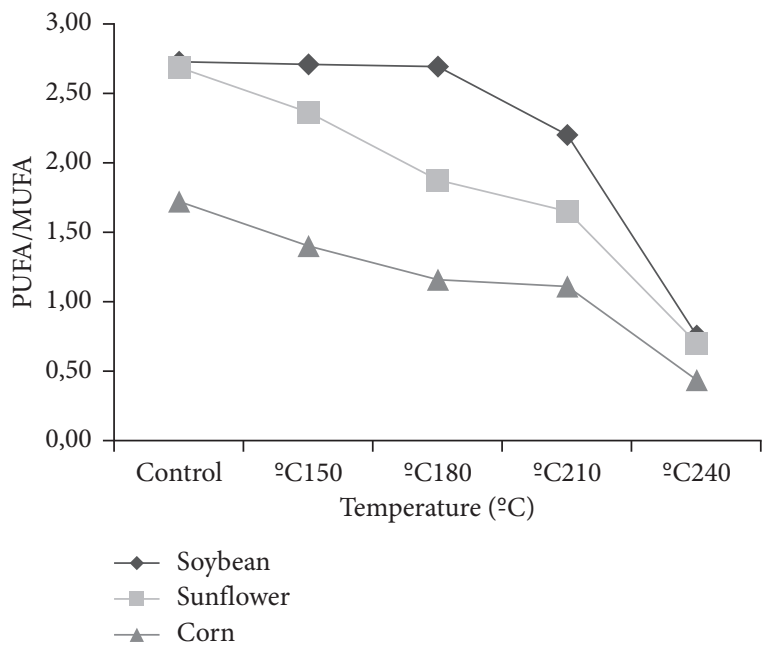

FIGURE 6: Evolution of PUFA/MUFA ratio of soybean, sunflower, and corn oils after 60 hours of heating. PUFA: polyunsaturated fatty acids and MUFA: monounsaturated fatty acids. degradation of fatty acids under the temperature effect and during a prolonged treatment time. This was confirmed by the work of $[27,54]$, who suggested that heated oils cause lipid oxidation involving oxidative destruction of the fatty acid chains. The present work shows an increase in free fatty acids represented by "Other FAs." This fraction accounted for $2.39,1.52$, and $1.71 \%$ of total fatty acids in fresh soybean, sunflower, and corn oils, respectively. While, these percentages reached $8.55,10.29$, and $7.11 \%$ of total fatty acids of soybean, sunflower, and corn oils, respectively, after $60 \mathrm{~h}$ of heating at $240^{\circ} \mathrm{C}$. Sunflower oil appeared to be most affected by the high temperature $\left(240^{\circ} \mathrm{C}\right)$ and extended processing time $(60 \mathrm{~h})$. These results are in agreement with the work of [55] which showed an increase in free fatty acid percentages with the frying time at $180^{\circ} \mathrm{C}$. In addition, some authors [56-58] have shown a significant formation of free fatty acids and a decrease in the peroxide value index in vegetable oils by prolonged microwave heating.

The results showed that the high temperature $\left(240^{\circ} \mathrm{C}\right)$ has a depressive effect on the levels of essential fatty acids 
proportional to the treatment duration and a very significant increase of the percentages of the following fatty acids: $\mathrm{C} 16$ : 0, C18: 0, C18: 1W9, C14: 0, C18: 1 11c. C20: 0. C22: 0 present a moderate increase; however, C22: 0 was completely absent from $T=180^{\circ} \mathrm{C}$ in corn oil.

The influence of the nature of the fatty acid composition on the stability and oxidation of the oil has already been demonstrated by several scientific studies [59]. Thermal treatment may affect the appearance of conjugated linoleic acid (CLA) isomers. The works of [60] showed that the CLA content of heated sunflower oil was higher when the oil was more heated $\left(1.3 \%\right.$ at $T=220^{\circ} \mathrm{C}$ against $0.2 \%$ at $\left.T=180^{\circ} \mathrm{C}\right)$. These heat treatments can also affect the trans-fatty acids content. Compared to fresh oil, the composition of transfatty acids changes after many uses as in frying oil [61].

3.8. The Treatment Effect on the Rate of Saturated Fatty Acids (SFAs). The major SFAs were represented by palmitic acid (C16: 0$)$ and stearic acid (C18: 0) in all oil samples. The rate of these fatty acids without treatment was $14.54,10.37$, and $12.31 \%$ for soybean, sunflower, and corn oil, respectively. The results showed stability in fatty acid composition during the heat treatment of oils at 150 and $180^{\circ} \mathrm{C}$, which is consistent with the results found by [62], who suggested that fatty acids such as C16: 0 or C18: 0 are more stable than unsaturated ones like C18: 1, C18: 2, and C18: 3. However, from the temperature of $210^{\circ} \mathrm{C}$, the levels of these fatty acids increase significantly and are about $50 \%$ of the initial levels after $60 \mathrm{~h}$ of treatment at $240^{\circ} \mathrm{C}$. In soybean and corn oil, palmitic acid was the major fatty acid among SFA; it ranged from $10 \%$ to $15 \%$ at 150,180 , and $210^{\circ} \mathrm{C}$.

Based on our results, it appears that the oils submitted to low temperatures $\left(150\right.$ and $\left.180^{\circ} \mathrm{C}\right)$ and different treatment times $(12,24,36,48$, and $60 \mathrm{~h})$ have some thermal stability and lipid structure preservation. However, the composition of these oils has been largely influenced by treatment at $210^{\circ} \mathrm{C}$ and $240^{\circ} \mathrm{C}$ and fatty acid levels vary with the duration of treatment. At these temperatures, it can be assumed that there was an inversely proportional relationship between SFA and essential fatty acids (C18: 2 and C18: 3).

\section{Statistical Analysis}

The statistical analysis provided was presented in Figures $1-3$. It was found that the maximum variation in fatty acid contents expressed by $\triangle C / C$ varies according to species, temperature, and duration of treatment (Figure 1). In fact, the maximum variation at $240^{\circ} \mathrm{C}$ is at $12 \mathrm{~h}, 48 \mathrm{~h}$, and $60 \mathrm{~h}$ respectively for sunflower, soybean, and corn oil, respectively. However, the maximum variation at $180^{\circ} \mathrm{C}$ is noted at $12 \mathrm{~h}$ for soybean and $60 \mathrm{~h}$ for sunflower and corn oil. It is clear that the highest values are always found in sunflower oil, whatever the temperature applied.

Figures 2 and 3 represent the variation in heating times, allowing the maximum variation in fatty acid content by species subjected to different temperatures. The results show that C18: $3 n-3$ levels are most affected at $240^{\circ} \mathrm{C}$ in sunflower and $\mathrm{C} 12: 0$ at $180^{\circ} \mathrm{C}$ in soybean after 12 hours of treatment.
After 24 hours and 36 hours of treatment, no changes were reported with the exception of C17: 0 (at $150^{\circ} \mathrm{C}$ ). After 48 hours, the maximum variation in soybean was found in the level of C17: 0 (at $150^{\circ} \mathrm{C}$ ) and C8: 0 (at $210^{\circ} \mathrm{C}$ and at $240^{\circ} \mathrm{C}$ ). After 60 hours of treatment, the major variations in soybean affect mainly C17: 0 (at $150^{\circ} \mathrm{C}$ ), for corn oil, C18: $3 n-3$ (at $180^{\circ} \mathrm{C}$ and $210^{\circ} \mathrm{C}$ ); C18: $1-9 t$ at $240^{\circ} \mathrm{C}$ and for sunflower $\mathrm{C} 18$ : $3 n-3$ at $180^{\circ} \mathrm{C}$ and $210^{\circ} \mathrm{C}$.

Under the action of the same temperature, the thermal behavior of fatty acids varies according to species. The maximum variation is found in C18: $3 n-3$ at $(0-12 \mathrm{~h})$, in C8:0 at (0-48 h), and in C18:9-1t at (0-60 h), respectively, in sunflower, soybean, and corn (Figure $3(\mathrm{~d})$ ). These results also confirm that sunflower is the most sensitive species, while corn is the most resistant to temperature variations.

\section{Conclusion}

The GC analysis of fatty acids of three fresh edible oils revealed the dominant presence of linoleic acid with a content of about 53, 54, and 55\% for corn, soybean, and sunflower, respectively. Oleic acid is present at 21,25 , and $30 \%$ respectively, for soybean, sunflower and, corn oil. The composition of these oils can be considered an important natural source of unsaturated omega- 6 fatty acids.

Stearic and palmitic acids are the main fatty acids among saturated fatty acids, the contents of these fatty acids together are close to $14 \%, 10 \%$, and $12 \%$ for respective soybean, sunflower, and corn oils. These oils that are high in polyunsaturated fatty acid content, are sensitive to heat. The heat treatment at different temperatures shows a variation in the composition of total fatty acids. This variation was due to a series of chemical transformations that can be oxidation reactions, isomerization, cyclization, polymerization, and other reactions.

Results suggest that oils subjected to $150^{\circ} \mathrm{C}$ and $180^{\circ} \mathrm{C}$ at different treatment periods have no significant effect on fatty acid composition; however, the composition of oils subjected to higher temperatures $\left(210\right.$ and $\left.240^{\circ} \mathrm{C}\right)$ was significantly affected. The rate of major saturated fatty acids (C16: 0 and C18: 0) continues to increase while that of unsaturated fatty acids continues to decrease according to the temperature and duration of treatment rise.

Essential fatty acids, which are more sensitive to thermal effects than the other fatty acids, decrease dramatically from $210^{\circ} \mathrm{C}$. Trans-fatty acids (18: $19 \mathrm{t}$; C18: $2 \mathrm{t}, \mathrm{t}$ ) were formed during the heating process, and the rates of these fatty acids depended mainly on the temperature reached, but also on the applied processing time. The increase in trans-fatty acids has been associated with a decrease of unsaturated fatty acids, suggesting that PUFA might be precursors of some TFA.

At $210^{\circ} \mathrm{C}$ or at $180^{\circ} \mathrm{C}$ with a long heating time, the oil will have deteriorated with chemical transformation and the appearance of smoke. At $240^{\circ} \mathrm{C}$, there was a browning of the oil, the appearance of stable foam, smoke, and incongruous odors. Consequently, the optimum temperature should not exceed $180^{\circ} \mathrm{C}$.

Depending on the fatty acid composition of these oils and their behavior as a function of thermal heating, it was 
concluded that sunflower oil is the most affected by the thermal effect, followed by soybean oil, while corn oil is the most resistant to the thermal process. Therefore, according to our study, it is advisable to use corn oil for frying and cooking. It should be noted that the increase of temperature and duration of the treatment seem to be the oxidation acceleration factors. Results suggested that thermal heating at high temperatures or/and at extended processing times generates significant production of trans isomers. Oxidative changes that occur during the heating process negatively affect the nutritional value as well as the sensory qualities of edible oils. Each oil has its characteristics of oxidation stability depending on the content and nature of fatty acids. Therefore, it is recommended to use a heating mode designed to limit local overheating that promotes fat degradation and to avoid heating too hard and too long, not to exceed 60 hours.

\section{Data Availability}

The data used to support the findings of this study are included in the article.

\section{Conflicts of Interest}

The authors declare that they have no conflicts of interest.

\section{Acknowledgments}

The authors are thankful for the technical support of Dr. Anis Ben Dhahbi and Mr. Ronan Corcuff.

\section{References}

[1] C. A. Curti, R. N. Curti, N. Bonini, and A. N. Ramón, "Changes in the fatty acid composition in bitter Lupinus species depend on the debittering process," Food Chemistry, vol. 263, pp. 151-154, 2018.

[2] F. Anwar, G. M. Kamal, F. Nadeem, and G. Shabir, "Variations of quality characteristics among oils of different soybean varieties," Journal of King Saud University Science, vol. 28, no. 4 , pp. 332-338, 2016.

[3] İ. Demiral, A. Eryazıc1, and S. Şensöz, "Bio-oil production from pyrolysis of corncob (Zea mays L.)," Biomass and Bioenergy, vol. 36, pp. 43-49, 2012.

[4] A. Nagao and M. Yamazaki, "Lipid of sunflower seeds produced in Japan," Journal of the American Oil Chemists Society, vol. 60, no. 9, pp. 1654-1658, 1983.

[5] H. M. Ibrahim, "Response of some sunflower hybrids to different levels of plant density," APCBEE Procedia, vol. 4, pp. 175-182, 2012.

[6] D.-S. Lee, B.-S. Noh, S.-Y. Bae, and K. Kim, "Characterization of fatty acids composition in vegetable oils by gas chromatography and chemometrics," Analytica Chimica Acta, vol. 358, no. 2, pp. 163-175, 1998.

[7] M. Chen, R. S. P. Rao, Y. Zhang, C. Zhong, and J. J. Thelen, "Metabolite variation in hybrid corn grain from a large-scale multisite study," The Crop Journal, vol. 4, no. 3, pp. 177-187, 2016.

[8] R. P. Mensink, P. L. Zock, A. D. Kester, and M. B. Katan, "Effects of dietary fatty acids and carbohydrates on the ratio of serum total to HDL cholesterol and on serum lipids and apolipoproteins: a meta-analysis of 60 controlled trials," The American Journal of Clinical Nutrition, vol. 77, no. 5, pp. 1146-1155, 2003.

[9] D. Mozaffarian, R. Micha, and S. Wallace, "Effects on coronary heart disease of increasing polyunsaturated fat in place of saturated fat: a systematic review and meta-analysis of randomized controlled trials," PLoS Medicine, vol. 7, no. 3, Article ID e1000252, 2010.

[10] J. Pavlisova, K. Bardova, B. Stankova, E. Tvrzicka, J. Kopecky, and M. Rossmeisl, "Corn oil versus lard: mtabolic effects of omega-3 fatty acids in mice fed obesogenic diets with different fatty acid composition," Biochimie, vol. 124, pp. 150-162, 2016.

[11] X. Li, G. Wu, F. Yang et al., "Influence of fried food and oil type on the distribution of polar compounds in discarded oil during restaurant deep frying," Food Chemistry, vol. 272, pp. 12-17, 2019.

[12] T. M. F. S. Vieira and M. A. B. Regitano-d'Arce, "Canola oil thermal oxidation during oven test and microwave heating," Lebensmittel-Wissenschaft und-Technologie-Food Science and Technology, vol. 34, no. 4, pp. 215-221, 2001.

[13] M. Musa Özcan, F. Y. Al-Juhaimi, I. A. Mohamed Ahmed, M. A. Osman, and M. A. Gassem, "Effect of different microwave power setting on quality of chia seed oil obtained in a cold press," Food Chemistry, vol. 278, pp. 190-196, 2019.

[14] M. S. Taipina, M. L. Garbelotti, L. C. A. Lamardo, J. S. Santos, and M. A. B. Rodas, "The effect of gamma Irradiation on the nutrional properties of sunflower whole grain cookies," 11th Int. Congr. Eng. Food ICEF11, vol. 1, 2011.

[15] N. Rodrigues, R. Malheiro, S. Casal, M. C. Asensio-Manzanera, A. Bento, and J. A. Pereira, "Influence of spike lavender (Lavandula latifolia Med.) essential oil in the quality, stability and composition of soybean oil during microwave heating," Food and Chemical Toxicology, vol. 50, no. 8, pp. 2894-2901, 2012.

[16] L. Giua, F. Blasi, M. S. Simonetti, and L. Cossignani, "Oxidative modifications of conjugated and unconjugated linoleic acid during heating," Food Chemistry, vol. 140, no. 4, pp. 680-685, 2013.

[17] X. Li, G. Wu, Y. Wu et al., "Effectiveness of the rapid test of polar compounds in frying oils as a function of environmental and compositional variables under restaurant conditions," Food Chemistry, vol. 312, Article ID 126041, 2020.

[18] E. N. Frankel, "Lipid oxidation," Progress in Lipid Research, vol. 19, no. 1-2, pp. 1-22, 1980.

[19] C. C. Akoh and D. B. Min, Food Lipids: Chemistry, Nutrition, and Biotechnology, CRC Press, Boca Raton, FL, USA, 2nd edition, 2002.

[20] H. Yoshida, N. Hirooka, and G. Kajimoto, "Microwave heating effects on relative stabilities of tocopherols in oils," Journal of Food Science, vol. 56, no. 4, pp. 1042-1046, 1991.

[21] T. Albi, A. Lanzón, A. Guinda, M. C. Pérez-Camino, and M. León, "Microwave and conventional heating effects on some physical and chemical parameters of edible fats," Journal of Agricultural and Food Chemistry, vol. 45, no. 8, pp. 3000-3003, 1997.

[22] M. M. Hassanein, S. M. El-Shami, and M. H. El-Mallah, "Changes occurring in vegetable oils composition due to microwave heating," Grasas y Aceites, vol. 54, pp. 343-349, 2003.

[23] R. S. Farag, F. M. Hewedi, S. H. Abu-Raiia, and G. S. ElBaroty, "Comparative study on the deterioration of oils by microwave and conventional heating," 2016. 
[24] A. Grandgirard, A. Piconneaux, J. L. Sebedio, S. F. O’Keefe, E. Semon, and J. L. Le Quére, "Occurrence of geometrical isomers of eicosapentaenoic and docosahexaenoic acids in liver lipids of rats fed heated linseed oil," Lipids, vol. 24, no. 9, pp. 799-804, 1989.

[25] G. Billek, "Health aspects of thermoxidized oils and fats," European Journal of Lipid Science and Technology, vol. 102, no. 8-9, pp. 587-593, 2000.

[26] J.-L. Sébédio, J.-M. Chardigny, and C. Malpuech-Brugère, "10-physiological effects of trans and cyclic fatty acids A2," in Deep Fry, pp. 205-228, AOCS Press, Boulder Urbana, IL, USA, 2nd edition, 2007.

[27] E. N. Frankel, "Chemistry of free radical and singlet oxidation of lipids," Progress in Lipid Research, vol. 23, no. 4, pp. 197-221, 1984.

[28] J. A. Rojo and E. G. Perkins, "Cyclic fatty acid monomer formation in frying fats. I. Determination and structural study," Journal of the American Oil Chemists' Society, vol. 64, no. 3, pp. 414-421, 1987.

[29] J. L. Sébédioo, J. Kaitaranta, A. Grandgirarda, and Y. Malkk, "Quality assessment of industrial prefried French fries," Journal of the American Oil Chemists Society, vol. 68, pp. 299-302, 1991.

[30] G. Márquez-Ruiz and M. C. Dobarganes, "Nutritional and physiological effects of used frying oils and fats A2," pp. 173-203, AOCS Press, Boulder Urbana, IL, USA, 2nd edition, 2007.

[31] S. Srivastava, M. Singh, J. George, K. Bhui, A. Murari Saxena, and Y. Shukla, "Genotoxic and carcinogenic risks associated with the dietary consumption of repeatedly heated coconut oil," British Journal of Nutrition, vol. 104, no. 9, pp. 1343-1352, 2010.

[32] Moreira, Deep Fat Frying: Fundamentals and Applications | Rosana G Moreira, Springer, Berlin, Germany, 1999.

[33] M. J. A. Williams, W. H. F. Sutherland, M. P. McCormick, S. A. de Jong, R. J. Walker, and G. T. Wilkins, "Impaired endothelial function following a meal rich in used cooking fat," Journal of the American College of Cardiology, vol. 33, no. 4, pp. 1050-1055, 1999.

[34] F. Soriguer, G. Rojo-Martínez, M. C. Dobarganes et al., "Hypertension is related to the degradation of dietary frying oils," The American Journal of Clinical Nutrition, vol. 78, no. 6, pp. 1092-1097, 2003.

[35] P. Møller and H. Wallin, "Adduct formation, mutagenesis and nucleotide excision repair of DNA damage produced by reactive oxygen species and lipid peroxidation product," $\mathrm{Mu}$ tation Research, vol. 410, pp. 271-290, 1998.

[36] J. Navarro, E. Obrador, J. Carretero et al., "Changes in glutathione status and the antioxidant system in blood and in cancer cells associate with tumour growth in vivo," Free Radical Biology and Medicine, vol. 26, no. 3-4, pp. 410-418, 1999.

[37] R. Brun and F. Rademakers, "Root-an object oriented data analysis framework," Nuclear Instruments and Methods in Physics Research Section A: Accelerators, Spectrometers, Detectors and Associated Equipment, vol. 389, no. 1-2, pp. 81-86, 1997.

[38] D. B. Min and T. H. Smouse, Flavor Chemistry of Fats and Oils, The American Oil Chemists Society, Champaign, IL, USA, 1985.

[39] F. Seniha Güner, Y. Yağc1, and A. Tuncer Erciyes, "Polymers from triglyceride oils," Progress in Polymer Science, vol. 31, pp. 633-670, 2006.
[40] M. C. M. Moya Moreno, D. Mendoza Olivares, F. J. Amézquita López, J. V. Gimeno Adelantado, and F. Bosch Reig, "Determination of unsaturation grade and trans isomers generated during thermal oxidation of edible oils and fats by FTIR," Journal of Molecular Structure, vol. 482-483, pp. 551-556, 1999.

[41] A. Grandgirard, F. Julliard, J. Prevost, and J. L. Sebedio, "Preparation of geometrical isomers of linolenic acid," Journal of the American Oil Chemists' Society, vol. 64, no. 10, pp. 1434-1440, 1987.

[42] R. L. Wolff and J.-L. Sébédio, "Characterization of $\gamma$-linolenic acid geometrical isomers in borage oil subjected to heat treatments (deodorization)," Journal of the American Oil Chemists' Society, vol. 71, no. 2, pp. 117-126, 1994.

[43] N. Achir, W. Kara, C. Chipeaux, I. Trezzani, and M. E. Cuvelier, "Effect of energy transfer conditions on the chemical degradation of frying oil," European Journal of Lipid Science and Technology, vol. 108, no. 12, pp. 999-1006, 2006.

[44] J. E. Hunter, "Dietary trans fatty acids: review of recent human studies and food industry responses," Lipids, vol. 41, no. 11, pp. 967-992, 2006.

[45] L. Bretillon, A. Roy, B. Pasquis, and J.-L. Sébédio, "Dietary cyclic fatty acids derived from linolenic acid do not exhibit intrinsic toxicity in the rat during gestation," Animal, vol. 2, no. 10, pp. 1534-1537, 2008.

[46] S. G. Sumnu and S. Sahin, Advances in Deep-Fat Frying of Foods, CRC Press, Boca Raton, FL, USA, 1ed edition, 2008.

[47] R. Uauy and A. D. Dangour, "Fat and fatty acid requirements and recommendations for infants of 0-2 years and children of 2-18 years," Annals of Nutrition and Metabolism, vol. 55, no. 1-3, pp. 76-96, 2009.

[48] T. Henri, S. Jacques, and L. Rene, "Twenty-two years screening for toxoplasmosis in pregnancy: Liege-Belgium," Scandinavian Journal of Infectious Diseases. Supplementum, vol. 84, pp. 84-85, 1992.

[49] A. Cherif, S. Boukhchina, and P. Angers, "GC-MS characterization of cyclic fatty acid monomers and isomers of unsaturated fatty acids formed during the soybean oil heating process," European Journal of Lipid Science and Technology, vol. 121, no. 5, Article ID 1800296, 2019.

[50] C. J. Palacios, A. Grandis, V. J. Carvalho, A. Salatino, and M. S. Buckeridge, "Isolated and combined effects of elevated $\mathrm{CO} 2$ and high temperature on the whole-plant biomass and the chemical composition of soybean seeds," Food Chemistry, vol. 275, pp. 610-617, 2019.

[51] J. L. Sebedio, A. Grandgirard, and J. Prevost, "Linoleic acid isomers in heat treated sunflower oils," Journal of the American Oil Chemists' Society, vol. 65, no. 3, pp. 362-366, 1988.

[52] M. Kasprzak, M. Rudzińska, R. Przybylski, D. Kmiecik, A. Siger, and A. Olejnik, "The degradation of bioactive compounds and formation of their oxidation derivatives in refined rapeseed oil during heating in model system," $L W T$, vol. 123, Article ID 109078, 2020.

[53] S. Y. Quek, B. S. Chu, and B. S. Baharin, "Commercial extraction of vitamin E from food sources," in Encycl. Vitam., V. R. E Preedy and R. R. Watson, Eds., pp. 140-152, Universiti Putra Malaysia, Seri Kembangan, Malaysia, 2007.

[54] C.-Y. Ng, X.-F. Leong, N. Masbah, S. K. Adam, Y. Kamisah, and K. Jaarin, "Reprint of "Heated vegetable oils and cardiovascular disease risk factors"," Vascular Pharmacology, vol. 62, no. 1, pp. 38-46, 2014.

[55] S. Naz, R. Siddiqi, H. Sheikh, and S. A. Sayeed, "Deterioration of olive, corn and soybean oils due to air, light, heat and deep- 
frying," Food Research International, vol. 38, no. 2, pp. 127-134, 2005.

[56] C. P. Tan, Y. B. Che Man, S. Jinap, and M. S. A. Yusoff, "Effects of microwave heating on changes in chemical and thermal properties of vegetable oils," Journal of the American Oil Chemists' Society, vol. 78, no. 12, pp. 1227-1232, 2001.

[57] C. P. Tan, Y. B. Che Man, S. Jinap, and M. S. A. Yusoff, "Effects of microwave heating on the quality characteristics and thermal properties of RBD palm olein," Innovative Food Science \& Emerging Technologies, vol. 3, no. 2, pp. 157-163, 2002.

[58] L. Cerretani, A. Bendini, M. T. Rodriguez-Estrada, E. Vittadini, and E. Chiavaro, "Microwave heating of different commercial categories of olive oil: part I. effect on chemical oxidative stability indices and phenolic compounds," Food Chemistry, vol. 115, no. 4, pp. 1381-1388, 2009.

[59] R. Parker, Q. Huang, and B. Tesfamariam, "Influence of 3hydroxy-3-methylglutaryl-CoA (HMG-CoA) reductase inhibitors on endothelial nitric oxide synthase and the formation of oxidants in the vasculature," Atherosclerosis, vol. 169, no. 1, pp. 19-29, 2003.

[60] P. Juanéda, S. B. de la Pérrière, J.-L. Sébédio, and S. Grégoire, "Influence of heat and refining on formation of CLA isomers in sunflower oil," Journal of the American Oil Chemists Society, vol. 80, pp. 937-940, 2003.

[61] Ovesen, Food Lipids: Chemistry, Nutrition, and Biotechnology, Food Science \& Technology) - Documents, Dokumen.Tips, New York, NY, USA, 3rd edition, 1998.

[62] K. Vijayaraj and A. P. Sathiyagnanam, "Experimental investigation of a diesel engine with methyl ester of mango seed oil and diesel blends," Alexandria Engineering Journal, vol. 55, no. 1, pp. 215-221, 2016. 